\title{
Trivium
}

Revue franco-allemande de sciences humaines et sociales - Deutsch-französische Zeitschrift für Geistesund Sozialwissenschaften

$30 \mid 2019$

La constitution au tournant des XXe et XXle siècles

\section{Das Verfassungsrecht im Zeichen der Wende hin zum Rechtsprechungskommentar - Methodenfragen}

\section{Alexandre Viala}

Traducteur : Olivier Joop

\section{OpenEdition}

Journals

Édition électronique

URL : http://journals.openedition.org/trivium/6666

DOI : $10.4000 /$ trivium.6666

ISSN : 1963-1820

Éditeur

Les éditions de la Maison des sciences de l'Homme

Référence électronique

Alexandre Viala, « Das Verfassungsrecht im Zeichen der Wende hin zum Rechtsprechungskommentar - Methodenfragen », Trivium [Online], 30 | 2019, online erschienen am 18 Dezember 2019, abgerufen am 08 September 2020. URL : http://journals.openedition.org/trivium/6666 ; DOI : https://doi.org/ $10.4000 /$ trivium. 6666

Ce document a été généré automatiquement le 8 septembre 2020

\section{(c) (i) (9)}

Les contenus des la revue Trivium sont mis à disposition selon les termes de la Licence Creative Commons Attribution - Pas d'Utilisation Commerciale - Pas de Modification 4.0 International. 


\title{
Das Verfassungsrecht im Zeichen der Wende hin zum Rechtsprechungskommentar - Methodenfragen
}

\author{
Alexandre Viala \\ Traduction : Olivier Joop
}

\section{NOTE DE L'ÉDITEUR}

Wir danken Herrn Alexandre Viala für die freundliche Genehmigung, diesen Artikel in deutscher Übersetzung zu publizieren.

Nous remercions M. Alexandre Viala de nous avoir accordé l'autorisation de traduire ce texte pour le présent numéro.

1 Unlängst noch wurde der Verfassungsrechtler im Kreis der Rechtswissenschaftler manchmal eher stiefmütterlich behandelt. Verfassungsrecht wurde nicht immer als Rechtsgebiet betrachtet, sondern, mit seinem Fokus auf die politische Verfasstheit der Institutionen und deren Akteure, eher als eine Art vertiefter journalistischer Arbeit wahrgenommen. Diese Zeit gilt nunmehr als vergangen, seit der Verfassungsrichter die Bühne betreten hat und Rechtsstreitigkeiten auch im Verfassungsrecht ausgetragen werden; damit ist auch der Verfassungsrechtler in den Augen seiner Kollegen in den rechtswissenschaftlichen Fakultäten zu einem vollwertigen Juristen avanciert. Mit eigenem Richter, eigenen Prozessen und eigener Rechtsprechung ausgestattet, kann der Gegenstand, für den sich der Verfassungsrechtler interessiert, nunmehr Anlass zu Rechtsprechungskommentaren geben. Der neue Schwung, den die Einführung der vorrangigen Frage zur Verfassungsmäßigkeit (question prioritaire de constitutionnalité, QPC) im Verfassungsprozessrecht gebracht hat, eröffnet dem Verfassungsrechtler die Möglichkeit, selbst zum Kommentator von Urteilen aufzusteigen, wie es damals, zur Zeit 
Maurice Haurious, den Verwaltungsrechtlern vergönnt war, nachdem mit dem CadotUrteil die Theorie des "Ministers als Verwaltungsrichter ${ }^{1}$ hinweggefegt und der Aufstieg der aufstrebenden Verwaltungsgerichtsbarkeit zum Gegenstand wissenschaftlicher Auseinandersetzung begünstigt worden war. Obgleich eingedenk der Tatsache, dass der Verfassungsrat »Entscheidungen« (décisions) und keine »Urteile» (arrêts) fällt, die Bezeichnung "Kommentator von Urteilen" in den Ohren mancher Puristen unangemessen klingen mag, soll sie hier gleichwohl Verwendung finden, bezeichnet sie doch für die Gemeinschaft der Verfassungsrechtler im Zeichen der vollen Verwirklichung des Rechtsstaats einen Wendepunkt - die Umstände ähneln durchaus jenen, die damals die Verwaltungsrechtler im Zeitalter des aufstrebenden Gesetzesstaats kannten.

2 Allgemein als Fortschritt verstanden, wirft die Angleichung des Verfassungsrechts an die anderen Rechtsdisziplinen gleichwohl eine Reihe von Fragen auf, die angesichts der Zweifel, die von manchem skeptischen Rechtswissenschaftler geäußert worden sind, beantwortet werden müssen. Die Bedeutung des Verfassungsprozesses in der akademischen Welt der Verfassungsrechtler, verstärkt durch die zunehmende Rechtsprechungstätigkeit des Verfassungsrates im Zuge der Einführung eines Verfahrens zur konkreten Normenkontrolle, habe das Potenzial, das Verfassungsrecht wesentlich zu verändern. Der nachdenkliche Blick mancher Autoren in Anbetracht der Wendung hin zur Verfassungsgerichtsbarkeit, die bereits vor Einführung der vorrangigen Frage zur Verfassungsmäßigkeit (QPC) begonnen hatte, äußerst sich darin, im Verfassungsprozess als Teilbereich des Verfassungsrechts nur die Spitze des Eisbergs zu sehen, wohingegen ihnen zufolge das wahre Verfassungsrecht, das in die Tiefe reichende, in den Handlungen der politischen Akteure zum Ausdruck komme, Handlungen, deren mehr oder weniger beständige Regelmäßigkeit zu dem führe, was in Bezug auf das anglo-amerikanische Recht als Verfassungskonvention oder Verfassungsüberlieferung bezeichnet wird. ${ }^{2}$ Nach Ansicht mancher entrückt die Überbetonung des Verfassungsprozesses den Verfassungsrechtler vom wahren Gegenstand seiner Disziplin, ebenso wie die Technisierung verfassungsrechtlicher Fragen durch den Verfassungsprozess ihn dazu verleitet, das Verfassungsrecht in einer der instrumentellen Vernunft - von der Heidegger befürchtete, sie breche den Menschen und stürze ihn in die Seinsvergessenheit ${ }^{3}$ - ähnlichen Weise zu vernachlässigen. Indem sie die Verfassungsrechtler dazu verleite, sich auf das Kommentieren der nunmehr erweiterten Rechtssprechungstätigkeit des Verfassungsrichters zu konzentrieren, verschärfe das Institut der vorrangigen Frage zur Verfassungsmäßigkeit diese Neigung zur Beschäftigung mit Verfassungsstreitigkeiten. Sie lege den Keim für eine und verführe zu einer übertrieben formalistischen Denkweise in einem Rechtsgebiet, das sich bis dahin durch eine gewisse Form von Vornehmheit auszeichnete. Mit nostalgischem Tonfall und einer Wortwahl, in der Trugbilder und Überzeichnungen so manches Mal gegenüber der Vernunft Oberhand gewinnen, wird beklagt, die edlen Verfassungsgrundsätze der Staatsorganisation - lange Zeit den Niederungen der Klagewut der Leute entzogen seien heutzutage zum Mittel findiger Advokaten für die Durchsetzung der subjektiven Rechte ihrer Klienten geworden.

3 Allerdings steht dies nicht im Zentrum des vorliegenden Beitrags. Ohne beanspruchen zu wollen, die Quelle des wahren Verfassungsrechts (zwischen Verfassungstext, Entscheidung des Verfassungsrichters und Verhalten der politischen Akteure) zu 
ermitteln - auf die Gefahr hin, in ein Übermaß an Dogmatismus zu verfallen -, soll es hier vielmehr um die Problematik gehen, unter welchen Voraussetzungen die Vorgehensweise des Verfassungsrechtlers, wenn er in die Rolle des Kommentators von Urteilen schlüpft, den Anspruch auf Wissenschaftlichkeit erheben darf. Wir beabsichtigen dabei nicht, diese normative Wende des Verfassungsrechts zu verbannen - schließlich gebührt ihr das Verdienst, diese Disziplin aus ihrem langen Dornröschenschlaf im Vergleich zu den anderen Rechtsdisziplinen gezogen zu haben -, sondern wir wollen nach der Vereinbarkeit dieser Wende zum Normativen mit den methodischen Anforderungen der Rechtswissenschaft fragen. Genauer untersucht werden soll die Fähigkeit dieses neuartigen Verfassungsrechts, den epistemologischen Bedingungen zu genügen, die notwendig sind, um in berechtigter Weise den Rang einer wissenschaftlichen Disziplin beanspruchen zu können. Was die Disziplin des Verfassungsrechts in der Gemeinschaft der Juristen, in deren Augen die Kommentierung der Rechtsprechung eine bedeutsame intellektuelle Übung darstellt, an Anerkennung gewonnen hat, könnte sie vielleicht in dem Maße an Wissenschaftlichkeit eingebüßt haben, in dem der Fallstrick einer »formalistischen Denkweise ", in die der Verfassungsrechtler durch seine Rechtsprechungsexegese geraten kann, die Gefahr mit sich bringt, dessen Tätigkeit zu einer bloßen Technik zu degradieren. ${ }^{4}$

4 Der Teil einer Rechtsdisziplin, der sich mit den Streitigkeiten in diesem Rechtsgebiet auseinandersetzt, führt nämlich den Juristen dazu, sich nur mit dem normativen Aspekt des »Rechts« zu befassen, mit anderen Worten mit dem, was gemäß den Bestimmungen einer Norm "sein soll«, auch wenn anerkannt wird, dass die in einem solchen Sinne verstandene Norm das Ergebnis der Auslegung durch den Richter ist. Nun bestehen allerdings erhebliche Zweifel an der Wissenschaftlichkeit einer Betrachtungsweise, die sich ausschließlich der normativen Seite des Rechts widmen würde. Dieser Verdacht wird durch eine einfache terminologische Beobachtung erhärtet: Als »Wissenschaften« werden ausdrücklich diejenigen Disziplinen tituliert, die sich mit dem, "was ist«, befassen, oder anders ausgedrückt, mit der Wirklichkeit der Dinge jenseits deren normativen Gehalts und parallel zu dem rein juristischen Blick auf einen besonderen Aspekt des sozialen Lebens. So wird das Verfassungsrecht durch eine von der "Politikwissenschaft" geleisteten Analyse ergänzt, ebenso wie das Verwaltungsrecht in der »Verwaltungswissenschaft«, das Strafrecht in der "Kriminologie« und das Zivilrecht in der "Rechtssoziologie» ihre Fortsetzung findet, wobei die letztgenannte Disziplin zwar nicht die Bezeichnung "Wissenschaft» im Namen trägt, aber unzweifelhaft eine darstellt: die Soziologie, zu der sie gehört, wird allgemein als Königsdisziplin der Sozialwissenschaften betrachtet. Demgegenüber wird bei den rechtlichen Fragen, die solchen ausdrücklich als wissenschaftlich betitelten Disziplinen entsprechen, so getan, als gehörten sie dem ungleich weniger edlen Bereich des rein Technischen an. ${ }^{5}$ Alles in allem scheint es, als gewönnen die Sozialwissenschaften ihren Status als wissenschaftliche Disziplinen ohne weiteres nur dann, wenn ihr Gegenstand, wie derjenige der Naturwissenschaften, empirischer Art ist, und verlören diesen Status im Gegenzug, wenn der Gegenstand rein normativer Art ist. Darin bestehe der Geburtsfehler dessen, was man als Rechtswissenschaft bezeichnet. Eine Schwäche, die sich verstärke, wenn diese Wissenschaft die in ihrem Bereich behandelten Fragen zu Streitsachen überbewerte.

5 Worin liegen die Gründe für diese Vermutung der Nicht-Wissenschaftlichkeit, die eine Rechtsdisziplin trifft, deren Interesse ausschließlich der normativen Seite ihres 
Forschungsgegenstandes gilt? In der vorliegenden Abhandlung wird die Auffassung vertreten, dass die Rechtswissenschaft, wenn sie darauf reduziert wird, nach dem "Sollen« $z u$ fragen, mit einem ernsten epistemologischen Problem konfrontiert ist, nämlich dass dann zwei Begriffe mit demselben Wort wiedergegeben würden, die bei Kelsen jeweils als Rechtsnorm und Rechtssatz (der die Norm beschreibt) bezeichnet werden. Der Kommentator von Urteilen steht genau vor der Herausforderung dieser semantischen Übereinstimmung der Norm, die in diesem Fall vom Gericht formuliert wird und Gegenstand des Kommentars ist, und des Rechtssatzes, der im Kommentar seinen Ausdruck findet. Dadurch ist die Grenze zwischen der Rechtswissenschaft und der von ihr untersuchten Welt des Rechts sehr dünn oder gar durchlässig. Als Wissenschaft aber kann nur die gelten, die Distanz gegenüber der von ihr untersuchten Welt wahrt. Denn ihr Forschungsobjekt ist nicht dasselbe wie ihr wissenschaftlicher Gegenstand. Die Welt, auf den sie ihren Blick richtet (ihr Forschungsobjekt), ist nur eine empirische Gegebenheit, von der aus die Wissenschaft ihren eigenen Gegenstand herausarbeiten muss (ihr Artefakt, wie die Biologen sagen würden). Seit Gaston Bachelards wohlbekanntem Werk $^{6}$ gilt es als gesicherte Erkenntnis, dass der Gegenstand einer Wissenschaft nicht vorgegeben ist. Er ist konstruiert, und die Wissenschaftlichkeit einer Disziplin lässt sich an ihrem konstruktivistischen Aspekt messen: Ist sie in der Lage, ihren Gegenstand aufzubauen? In dieser pragmatischen Fähigkeit beruht die Wissenschaftlichkeit jedes akademischen Diskurses. Die Herausforderung ist schwer $\mathrm{zu}$ meistern, wenn die Welt, für die sich eine Wissenschaftsdisziplin interessiert, eine Welt voller normativer Aussagen ist, deren Beschreibung nur zu Aussagen mit einem sterilen analogen semantischen Gehalt führt. Was Kelsen den Rechtssatz nannte, ist somit nur ein klägliches Werkzeug, das für den Juristen keinerlei Hilfe bietet, wenn dieser seinen Gegenstand konstruieren will.

6 Der Verfassungsrechtler, heutzutage ganz ergriffen von der Begeisterung für Rechtsprechungskommentare, kann diese auf seiner Disziplin lastende Vermutung der Nicht-Wissenschaftlichkeit nur widerlegen, wenn es ihm gelingt, beim Aufbau seines wissenschaftlichen Gegenstandes zwei Arten von Anforderungen Genüge zu leisten. Für den Kommentator von Urteilen besteht die erste darin, einen höheren Grad von Abstraktheit im Vergleich zu den Aussagen der Gerichte, deren Entscheidungen er kommentiert, zu erreichen. Die Aussagen aus den konkreten Gerichtsentscheidungen sind nicht das Artefakt der Rechtswissenschaft, denn sie liegen bereits vor, der Jurist kann sie in ihrer ganzen Kontingenz einfach lesen. Der wissenschaftliche Gegenstand, mit dem sich der Verfassungsrechtler beschäftigt, ist hingegen eine pure Konstruktion, das Ergebnis einer gegenüber den Umständen des Einzelfalls eingenommenen Distanz, die darin besteht, rechtliche Kategorien zu schaffen, deren Allgemeingültigkeit und Abstraktheit von der konstruktiven Dimension der Vorgehensweise des Verfassungsrechtlers Zeugnis geben (I). Die zweite Bedingung, um den konstruktivistischen Anspruch zu erfüllen, dem auch die gegenwärtige Begeisterung für den Verfassungsprozess sich nicht $z u$ entziehen vermag, beruht darin, den Grundsatz der Kausalität zu verwenden, auf den seit dem 18.Jahrhundert jede wissenschaftliche Disziplin zurückgreift, um ihren Gegenstand aufzubauen. Kausalitätszusammenhänge sind das Werkzeug schlechthin, mit dem der Wissenschaftler selbst den Gegenstand seiner Wissenschaft konstruiert, und zwar aufgrund der bloßen Tatsache, dass der Grundsatz der Ursächlichkeit weder in der Natur (wenn es um Naturwissenschaften geht) noch im Recht (wenn es um die Rechtswissenschaft geht) liegt. Es gibt keinen Grund, warum der Kommentator 
verfassungsgerichtlicher Entscheidungen in seiner Funktion als Rechtsgelehrter auf ein derartiges Requisit verzichten sollte. Daher ist es bedeutsam, eine allgemeine Theorie des Verfassungsrechts aufzubauen, die ihren Gegenstand in Kausalzusammenhängen begreift (II).

\section{Allgemeingültigkeit als Hauptaufgabe der Verfassungswissenschaft}

7 Die von Gaston Bachelard formulierte epistemologische Anforderung, der sich Juristen nicht entziehen dürfen, wenn sie den Anspruch erheben, wissenschaftlich vorzugehen, wird von deren Kollegen in anderen Wissenschaftsdisziplinen ganz natürlich befolgt. Gewinnt die behandelte empirische Gegebenheit nicht die Form eines Normsetzungsdiskurses, erfolgt deren wissenschaftliche Beschreibung und Benennung im Rahmen eines eigenständigen akademischen Diskurses, dessen Inhalt zwangläufig aus einer Konstruktion hervorgeht. Um im Bereich des Rechts eine solche Konstruktion $\mathrm{zu}$ erreichen, muss der Jurist im Vergleich zu einem Nicht-Juristen eine besondere, wenn nicht verstärkte Anstrengung leisten, denn die normativen Aussagen, die seiner Betrachtung unterliegen, weisen die Besonderheit auf, ihm die Illusion zu vermitteln, sie seien bereits der wissenschaftliche Gegenstand. Angesichts dieser Lage muss der Jurist auf die Methode der Theoriebildung zurückgreifen, die darin besteht, jenseits einer bloßen Wiederholung der normativen Aussagen, die das Rohmaterial seiner Betrachtungen bilden, Rechtskategorien $\mathrm{zu}$ konstruieren, die geeignet sind, das Durcheinander dieser Aussagen in etwas Begreifbares zu verwandeln (A).

8 Ein solches Unterfangen bewirkt, über die Erkenntnisse aus der empirischen Beobachtung dieser Aussagen hinaus, die Herausbildung einer Reihe von Konzepten, die zur kontingenten Sphäre des positiven Rechts hinzutreten. Es geht darum, die Verschiedenartigkeit der untersuchten Aussagen des positiven Rechts herunterzubrechen auf ein einheitliches System, das wiederholbar und auch auf Aussagen anwendbar ist, die mit den konkret untersuchten normativen Aussagen vergleichbar sind. Daher stellt für den Verfassungsrechtler der rechtsvergleichende Blick eine große Bereicherung dar, der es ihm ermöglicht, auf der Grundlage der Befassung mit diesem oder jenem spezifischen positiven Recht für den Gegenstand seiner Wissenschaft ein Modell zu entwickeln (B).

\section{A. Die Vorteile der theoretischen Herangehensweise: die Konstruktion des wissenschaftlichen Gegenstands}

Das wesentliche Erkenntnishindernis, das die Rechtswissenschaft im Besonderen zu überwinden hat, ist folgendes: Während im Allgemeinen die vom Wissenschaftler beschriebene Welt eine Welt ohne Signifikanten ist - eine Welt, die vom Wissenschaftler souverän benannt wird -, weist die vom Juristen beschriebene Welt, die ausschließlich auf einem normativen Diskurs beruht, die Besonderheit auf, dass sie sich selbst als Welt des Rechts bezeichnet. In einem solchen Diskurs geht es um Aussagen, deren Urheber den Anspruch erheben, Normen zu erzeugen, d. h. Aussagen mit einer normativen Bedeutung, die dem Rechtswissenschaftler, der sich mit ihnen befasst, die Illusion vermitteln, der Gegenstand seiner Wissenschaft sei bereits konstituiert. Daraus ergibt sich, dass der Aussagegehalt des rechtswissenschaftlichen 
Diskurses fast identisch ist mit demjenigen des Diskurses des Rechts selbst. Wenn in Frankreich ein verfassungsrechtliches Lehrbuch die Aussage enthält, »der Präsident der Republik wird alle fünf Jahre neu gewählt«, so verweist es auf keinen anderen Sachverhalt als denjenigen, der von der französischen Verfassung vorgegeben wird, doch im Unterschied $\mathrm{zu}$ dieser, die einen Willen und eine Handlungsanweisung ausdrückt, vermittelt jenes eine Information, ein Stück Wissen ... Andere Zwecke, doch dieselbe Aussage.

Die Folge aus dieser semantischen Übereinstimmung ist beachtlich: Das Recht ist jene einzigartige Disziplin, bei der der Wissenschaftler bei der Schaffung seines wissenschaftlichen Gegenstandes erhebliche Hindernisse zu überwinden hat, die daher rühren, dass zwischen ihm und der Welt, die er untersuchen soll, eine besondere, fast »inzestuöse« Beziehung besteht. Der Aufbau eines wissenschaftlichen Gegenstandes wird ihm erschwert, da eine übermäßige Nähe vorliegt zwischen der Welt, die seine Neugier entfacht, und den Ausdrucksmitteln zur Beschreibung dieser Welt - beiden ist ihr diskursiver Charakter eigen. Einem Insektenkundler ist dieses Problem fremd - eine von ihm untersuchte Fliege hat sich selbst nie als "Drosophila» bezeichnet - und es steht ihm frei, dieses Insekt zu vergegenständlichen, indem er ihm die Bezeichnung "Drosophila« gibt. Auch ein Soziologe, dem die von ihm untersuchten menschlichen Gruppen nie vorgegeben haben, sie seien der "Psychologie der Massen« unterworfen gewesen, als sie die Machtergreifung eines Diktators guthießen, kann den Gegenstand seiner Wissenschaftsdisziplin eigenständig benennen - und erschaffen. ${ }^{7}$

11 Bereits Kelsen stellte fest: »Eine Pflanze kann dem sie wissenschaftlich bestimmenden Naturforscher nichts über sich selbst mitteilen. Sie macht keinen Versuch, sich selbst naturwissenschaftlich zu erklären «. ${ }^{8}$ Für den Juristen ist die Situation eine andere; er muss sich damit auseinandersetzen, dass die »Dinge«, die er bezeichnen soll, sich selbst als Rechtsakte bezeichnen. Die Rechtswissenschaft mit ihrem normativen Gegenstand steht einem Problem gegenüber, das den empirischen Wissenschaften - beispielsweise den Naturwissenschaften oder den nicht normativen Sozialwissenschaften wie der Soziologie - mit ihrem "stummem « Gegenstand zwangsläufig fremd ist. ${ }^{9}$ Kelsen schreibt weiter: "Die das Recht begreifende Erkenntnis findet mitunter schon eine rechtliche Selbstdeutung des Materials vor, die der von der Rechtserkenntnis zu leistenden Deutung vorgreift. « ${ }^{10}$ Die Rationalisierung dieses Materials ist bereits erfolgt, bevor der Rechtswissenschaftler eingreift. Diesem fällt es mithin schwer, der Anleitung Bachelards Folge zu leisten, wonach Wissenschaft ihren Gegenstand durch dessen Benennung zu konstruieren hat. Sicherlich konstruiert der Wissenschaftler den wissenschaftlichen Gegenstand nicht aus dem Nichts heraus, er ist zunächst Betrachter einer Unordnung, die ihm zufällt und die er jedoch verständlich zu machen hat. In dieser Rationalisierung liegt der Vorgang, stummen Dingen einen Namen zu geben; dadurch leistet der Wissenschaftler Konstruktionsarbeit. Was sofort die Frage nach sich zieht: Wie kann einer Disziplin Wissenschaftlichkeit zuerkannt werden, die wissenschaftliche Diskurse in Bezug auf Aussagen produziert, die aufgrund der Tatsache, dass sie bedeutungstragende Aussagen darstellen, die Aussichten des Wissenschaftlers schmälern, eigenständig seinen Gegenstand zu konstruieren? Anders formuliert: Wie kann der Jurist die unverzichtbare Aufgabe erfüllen, rational Ordnung in das Chaos zu bringen; nur dann ist eine konstruktive Leistung überhaupt feststellbar, denn das zu ordnende Chaos besteht aus signifikanten Aussagen, welche die Illusion vermitteln, sich zum wissenschaftlichen Gegenstand zu erheben, wodurch die Gefahr 
besteht, dass sie den Wissenschaftler beeinträchtigen und daran hindern, den Bachelard'schen Anforderungen gerecht zu werden.

Die Antwort liegt in der theoriebildenden Herangehensweise. Dabei werden jenseits einer bloßen Wiederholung der normativen Diskurse, die illusorischerweise als wissenschaftlicher Gegenstand der Rechtswissenschaft erscheinen könnten, allgemeine Kategorien entwickelt, die es erlauben, die Unordnung dieser mit normativem Anspruch auftretenden Aussagen zu ordnen, zu erkennen und zu begreifen, sodass es anschließend möglich ist, daraus gemeinsame und verallgemeinerbare unveränderliche Größen (Gesetzmäßigkeiten) zu ziehen - was eine wissenschaftliche Vorgehensweise auszeichnet. Der Gegenstand der Rechtswissenschaft ist das Ergebnis dieser das Chaos ordnenden Vorgehensweise, die ein theoretischer Ansatz ist, da sie darauf zielt worauf bereits die Herkunft des Begriffes »Theorie« schließen lässt - das in einer Sache verborgene Göttliche (theion) zu erblicken (orao), d. h. die Logik beziehungsweise den Geist (logos), der diese Sache beseelt und leitet, zu entdecken: theion orao, »ich sehe das Göttliche«. Dieser vom Wissenschaftler geschaffene Gegenstand zur Erklärung dieser Unordnung nimmt die Gestalt sogenannter Rechtskategorien an (im hier betrachteten Bereich handelt es sich um Kategorien verfassungsrechtlicher Art). Das Göttliche zu erkennen war bereits von der griechischen Antike bis zur Scholastik des Mittelalters die dem Gelehrten zugeschriebene Bestimmung. Für die antiken Denker war die Wissenschaft kein Hilfsmittel des Fortschritts, sondern Selbstzweck, mit dem ausschließlich spekulativen Ziel, im Kosmos die Harmonie eines göttlichen Plans aufzuspüren, der jedem seinen unverrückbaren Platz in der göttlichen Ordnung zuweist. In diesem Sinne war eine Theorie nichts anderes als eine Metaphysik des Kosmos. Vor diesem historischen Hintergrund wäre es verständlich, wenn nach der Wende hin zu empirischer Wissenschaft seit dem 17. Jahrhundert dem idealistisch geprägten Begriff der »Theorie« mit Misstrauen begegnet würde. Doch obgleich der Theoretiker unserer Tage, im Gegensatz zu demjenigen der Antike, nicht mehr danach strebt, das im engeren Sinne Göttliche in den Dingen zu erkennen, besteht seine Vorgehensweise noch immer darin, die unsichtbaren Facetten dieser Dinge freizulegen, indem er sie einer logischen und systematischen Darstellung unterwirft. Gemeinsam ist dem antiken theoretischen Vorgehen und dem unserer Tage allerdings, dass sie stets darin besteht, sich von den sinnlich fassbaren Dingen selbständig zu machen und eine Darstellung der realen Welt mittels allgemeiner, universeller und gleichmäßiger Gesetze anzubieten. Seitdem sich die moderne Erkenntniswissenschaft etabliert hat, ist ein weltliches Verständnis dieses Begriffes üblich, weit entfernt von einer metaphysischen Betrachtungsweise. Allerdings geht es auch weiterhin darum, durch Theoriebildung diejenige Seite der Dinge zu erfassen, die bei einer rein sinnlichen Herangehensweise verborgen bleiben. Die Theorie sucht noch immer nach dem Göttlichen, jedoch verstanden als die verborgene Seite der Wirklichkeit.

Der Jurist, dessen Erkenntnisschwierigkeiten sich aus der semantischen Nähe zwischen dem präskriptiven Diskurs der rechtssetzenden Organe und dem deskriptiven Diskurs auf der Meta-Ebene ergeben, mit dem das Recht erforscht und gelehrt wird, ist von dieser theoriebildenden Vorgehensweise umso abhängiger, als es ihm unmöglich ist, den Gegenstand seiner Wissenschaft nur aufgrund einer rein empirischen Vorgehensweise zu konstruieren. Ohne eine theoretische Herausarbeitung bleibt der Gegenstand unsichtbar. Diese Herangehensweise ist das Lebenselixier der Rechtswissenschaft, es verhindert, dass der Jurist in einen fruchtlosen Diskurs ohne Erkenntnisgewinn verfällt: Da zweckbedingt ein Unterschied zwischen den 
Äußerungen eines Richters, der ein Urteil spricht, und eines Kommentators dieses Urteils besteht - der Richter legt den Inhalt einer Vorschrift aus, der Verfasser eines Kommentars sucht die Bedeutung hinter einer Darstellung -, führt der Gebrauch derselben Begriffe in der Sprache des Richters und des Verfassers eines Rechtsprechungskommentars unweigerlich dazu, dass ein solcher Kommentar lediglich zu einem trockenen Bericht des ergangenen Urteils verkommt, wenn dessen Verfasser auf eine theoretische Auseinandersetzung mit dem Urteil verzichtet. Das Heil liegt für den Juristen somit darin, sich zu überwinden und eine scharfe Trennlinie zu ziehen zwischen seinem eigenen Sprachgebrauch und dem des Richters. Wenn beispielsweise der Jurist die Auslegungsvorbehalte in den Entscheidungen des Verfassungsrates mit dem Begriff der »dritten Entscheidungsart» qualifiziert und dadurch nahelegt, Auslegungsvorbehalte nicht als Ausdruck einer Rechtsprechungstechnik, sondern als neuartigen Entscheidungstyp anzusehen, der die klassische Einteilung verfassungsgerichtlicher Urteilssprüche in Verfassungswidrigkeitserklärungen und Verfassungskonformitätserklärungen erweitert, schafft er gewissermaßen seinen wissenschaftlichen Gegenstand, insoweit der Verfassungsrat selbst nie diesen Begriff für die Auslegungsvorbehalte in seinen Entscheidungen verwendet hat. ${ }^{11}$ Die gleiche Beobachtung gilt, wenn ein Rechtswissenschaftler z. B. eine Dissertation zum Thema "Der Konsequentialismus in der Rechtsprechung des Verfassungsrates « verfasst. ${ }^{12} \mathrm{Er}$ konstruiert seinen Gegenstand ausgehend von einer sorgfältigen Lektüre der verfassungsgerichtlichen Entscheidungen, wobei das Verfassungsgericht selbst nie eine derart präzise und wissenschaftliche Terminologie verwendet hat, um solche seiner Entscheidungen $\mathrm{zu}$ bezeichnen, in denen ein aufmerksamer Leser einen konsequentialistischen Gedankengang aufzeigen kann. „Eine Dissertation zu verfassen bedeutet, einen Gegenstand zu entwickeln.« Diesen ersten methodischen Rat sollte jeder Doktorvater seinem Doktoranden zu Anfang von dessen Forschungsarbeit geben.

Zwischen dem Richterspruch und den Ausführungen des Juristen auf der Meta-Ebene muss es einen Unterschied geben, ohne den ansonsten die Rechtswissenschaft nichts weiter täte als eine Reihe von Rechtssätzen zusammenzutragen, die in empirischer Weise lediglich den Inhalt der von ihnen beschriebenen Normen wiedergeben. Den epistemologischen Gewinn, der sich aus diesem Unterschied ergibt, hat Michel Troper wie folgt beschrieben:

»Der Rechtssatz: >Der Präsident der Republik wird in allgemeiner und unmittelbarer Wahl für eine Amtszeit von fünf Jahren gewählt « ist richtig, wenn das positive Recht eine Norm enthält, die bestimmt: ১Der Präsident der Republik wird in allgemeiner und unmittelbarer Wahl für eine Amtszeit von fünf Jahren gewählt‘. Doch betrachten wir die allgemeinen rechtstheoretischen Sätze, die besagen: >Auslegung ist eine Tätigkeit des Willens` und >Die Rechtsordnung kennt keine Lücke`. Diese Sätze ergeben sich nicht unmittelbar aus einer Betrachtung des positiven Rechts. Sie beschreiben keine Rechtsnorm. Man kann sie, indem man das positive Recht jedes Landes nach einer Norm gleichen Inhalts durchforsten würde, weder bestätigen noch widerlegen, denn eine derartige Norm existiert nicht. Sie umschreibt darüber hinaus auch keine Aussage vergleichbaren Inhalts aus der Sprache des positiven Rechts. Es ist durchaus denkbar, dass die entsprechende Sprache keine Aussage über die Art der Auslegung enthält. Oder umgekehrt kann es sein, dass sie eine solche Aussage enthält, aber mit entgegengesetztem Inhalt. Daraus ergäbe sich allerdings keineswegs, dass die betrachteten allgemeinen rechtstheoretischen Sätze keine Gültigkeit besitzen «. ${ }^{13}$

Das ideale Vorgehen besteht somit darin, jenseits einer bloßen Wiederholung der normativen Aussagen allgemeingültige Kategorien zu schaffen, welche die Unordnung 
des positiven Rechts strukturieren und in Bezug auf dieses Konstanten herausarbeiten sowie Erklärungsschemata und Begriffe entwickeln. Ein im erkenntnistheoretischen Sinn idealistisches Vorgehen ist dafür bestens geeignet. Unter Idealismus ist hier nicht zu verstehen, dass es darum ginge, unter dem Banner der Rechtswissenschaft ein Ideal vorzuschreiben, wie dies die naturrechtlichen Theorien tun, sondern die Idee hinter einer Sache zu ergründen. So verstanden, beinhaltet jedes theoriegestützte Vorgehen eine idealistische Komponente - einer der ältesten Grundsätze der Theorie des Erkennens. Er geht, wie noch zu sehen sein wird, auf Platon und das Höhlengleichnis zurück, das im westlichen Denken die Herausbildung des wissenschaftlichen Geistes und die Vorliebe für abstraktes Denken anstelle des gewöhnlichen Wissens über unmittelbar erlebte Dinge markiert, die in ihrer Zufälligkeit und Kurzlebigkeit lediglich erlauben, sich eine Meinung über sie zu bilden. ${ }^{14}$ Gemäß dieser idealistischen Betrachtungsweise liegt die Wahrheit nicht in den zu einer illusorischen Welt voller Darstellungen gehörenden sinnlich erfahrbaren Dingen: Hinter der sichtbaren und sinnlich erfahrbaren Welt, die nach Ansicht der Juristen die normativen Aussagen der rechtssetzenden Akteure darstellen, verbirgt sich eine andere Welt, eine wahre Welt, die sich jeglicher unmittelbaren Wahrnehmung entzieht und sich nur nach kritischer Erkenntnisgewinnung offenbart. Eine intelligible Welt, gebildet aus Kategorien, die nur dann einen erkenntnistheoretischen Wert besitzen, wenn sie nach Allgemeingültigkeit und Zeitlosigkeit tendieren. Wissenschaft besteht darin, Modelle zu bilden und dadurch Licht in das Dickicht der ganzen Vielfalt der Wirklichkeit zu bringen, dass sie diese mittels einer allgemeingültigen Gestalt abbildet. Darin liegt, insbesondere im Bereich des Verfassungsrechts, auch der wertvolle Beitrag der Rechtsvergleichung.

\section{B. Der Beitrag rechtsvergleichender Betrachtungen: die Modellierung des wissenschaftlichen Gegenstands}

Bevor der Jurist als Antwort auf die Suche nach seinem wissenschaftlichen Gegenstand theoretische Aussagen erzeugen kann, muss er selbstverständlich zunächst die normative Sachlage empirisch untersuchen, die er anschließend gedanklich erfassen und Kategorien zuordnen will. Ein solches wissenschaftliches Protokoll ist insbesondere für den rechtsvergleichenden Juristen das probate Mittel. Der Zweck der Rechtsvergleichung besteht darin, durch eine Betrachtung der Vielfalt der nationalen positiven Rechtsordnungen Erkenntnisse über das Wesen des Rechts im Allgemeinen zu erlangen. Sie stellt aufgrund ihrer Eigenständigkeit und Freiheit im Stadium des Aufbaus des wissenschaftlichen Gegenstands das archetypische methodische Vorgehen des theoriegestützten Gedankengangs dar.

Unter epistemologischen Gesichtspunkten ist die Rechtsvergleichung deshalb von Interesse, weil ihr Diskurs niemals die bloße Wiederholung einer normativen Aussage des positiven Rechts darstellt. Sie ist nichts anderes als eine Teildisziplin bzw. ein Beitrag zur Rechtswissenschaft. ${ }^{15}$ Es gibt zwar etwa das französische Recht, das britische Recht, das EU-Recht oder auch das Völkerrecht als Rechtsordnung, doch kein Bürger der Welt kann je den Vorschriften des vergleichenden Rechts unterworfen sein. Das vergleichende Recht existiert nur als akademische Disziplin, die auf der Grundlage der Untersuchung der positiven Rechtsordnungen induktiv Invarianten und weitere universelle Gesetzmäßigkeiten ausmachen will, mit denen sich zwischen diesen verschiedenen Rechtspraxen Gemeinsamkeiten oder auch Unterschiede aufzeigen 
lassen, um so das Wesen des Rechts im Allgemeinen zu erfassen. Das vergleichende Recht ist kein positives Recht, es kann nichts anderes sein als Wissenschaft. Ausgehend von einer wissenschaftlichen Untersuchung der nationalen Rechtsordnungen ermöglicht es, ein invariantes Modell für das Verständnis des positiven Rechts zu erstellen, ein Modell, das auf alle individuellen und kontingenten Rechtsordnungen anwendbar ist. Es ist daher ein rein theoretisches Konstrukt.

Dabei ist dem rechtsvergleichenden Juristen nicht vollständig bewusst, dass er eine Arbeit theoretischer Art leistet und den Gegenstand seiner Wissenschaft schafft. Wie die meisten seiner Kollegen aus den anderen Rechtszweigen vermittelt er den Eindruck, dass er sich selbst als Jurist betrachtet, der das positive Recht empirisch beschreibt, ohne dabei konstruktiv tätig zu sein. Damit ist das einzige Monopol, das der »Theoretiker« zu Recht für sich beanspruchen kann - wenn ihm die normalerweise von allen Juristen geteilte Fähigkeit verwehrt bleibt, Rechtskategorien zu erschaffen -, das Bewusstsein dafür, dass jeder in seinem eigenen Bereich $\mathrm{zu}$ dieser Kategorisierungsarbeit beiträgt. So ist die Fähigkeit zur Reflexivität oder Distanznahme in Bezug auf das eigene Juristenhandwerk der ausschließlich denjenigen vorbehaltene Beitrag, die für sich die Bezeichnung »Theoretiker« beanspruchen. Von dieser Warte aus handelt es sich bei der Rechtstheorie, die von "Rechtstheoretikern" entwickelt wird, um jenes Bestreben nach Reflexivität, jenen Diskurs auf der Meta-Metaebene, der zum Ziel hat, darüber zu reflektieren, in welchem Maße der Metadiskurs der Rechtswissenschaft jene theoretischen Aussagen und allgemeingültigen Kategorien hervorzubringen vermag, ohne die eine Rechtswissenschaft überhaupt nicht möglich ist. Der "Theoretiker", der für sich selbst diese Bezeichnung in Anspruch nimmt, ist somit jener Jurist, der - vergleichbar den Meistern des Zweifelns aus anderen Wissenschaftsfeldern - in objektiver Weise die Lage seiner Juristenkollegen analysiert und sich bemüht, diese das Ausmaß des höchst subjektiven und konstruktiven Charakters ihrer Kategorisierungsarbeit erkennen zu lassen. Es sind in erster Linie Rechtsepistemologen, die eine solche Distanznahme in Bezug auf die Art und Weise leisten, mit der alle Rechtszweige behandelt werden, insbesondere der Wissenskorpus des Verfassungsrechts.

19 Für den »Theoretiker", der auf dieser Meta-Metadiskursebene agiert, ist es in der Tat lohnend, den Grad theoretischen Nachdenkens in der französischen und ausländischen Rechtslehre zu ermessen, insbesondere im Bereich der Typenlehre politischer Systeme, der Entwicklung von Modellen der Verfassungsgerichtsbarkeit oder auch der Definition des Verfassungsbegriffs, in Bezug auf den vor dem Hintergrund der Europäisierung und Internationalisierung des Konstitutionalismus eine notwendige Anpassungsentwicklung feststellbar ist. Auch wenn der rechtsvergleichende Jurist, insbesondere der verfassungsvergleichende Jurist, den von ihm geführten Diskurs nicht immer explizit als theoretischen deklariert, so folgt er doch unausgesprochen einem theoretischen Denkmuster, und zwar aufgrund der schlichten Tatsache, dass er ganz und gar den Gegenstand seiner Wissenschaft kreiert, wenn er auf der Basis einer Analyse der positiven Rechtsordnungen systematische Kategorien induziert. Als Louis Favoreu auf der Grundlage eines Vergleichs der Normenkontrollverfahren in den Vereinigten Staaten und in Europa die Unterscheidung zwischen dem Begriff des Obersten Gerichtshofs und dem Begriff des Verfassungsgerichts einführte, schuf er ein Modell der verschiedenen Arten von Verfassungsgerichtsbarkeit und erwies sich als Rechtstheoretiker, auch ohne diese Bezeichnung für sich in Anspruch zu nehmen. ${ }^{16}$ Die Schaffung dieser Kategorien, die es der Rechtswissenschaft erlaubt, ihren Gegenstand 
zu konstruieren und gleichzeitig ihren Status als Wissenschaft sichert, gehört zu den epistemologischen Gepflogenheiten des Verfassungsrechtlers, wenn er beispielsweise eine Typenlehre der politischen Systeme erstellt (parlamentarisches System/ Präsidialsystem), deren Bezeichnungen in dieser Form in den unterschiedlichen von ihm analysierten Verfassungen nicht vorkommen. Dieser konstruktivistische - und damit theoretische - Ansatz stellt somit ein Indiz dafür dar, dass es sich bei der Rechtstheorie weniger um eine spezifische Disziplin als vielmehr um einen heilsamen methodologischen Reflex handelt. Viele Juristen sind gleichzeitig Theoretiker, ohne sich dessen völlig bewusst zu sein. Die Lehrbücher von Maurice Hauriou, Léon Duguit und Raymond Carré de Malberg, ${ }^{17}$ die zu Beginn des 20. Jahrhunderts die ersten bedeutenden Kategorien des öffentlichen Rechts entwickelt haben, stellen in vieler Hinsicht genauso rechtstheoretische Werke wie verfassungsrechtliche Abhandlungen dar. Und die Theorien, die sie entwickelten - und die noch im 21. Jahrhunderts ernstgenommen werden -, sollten über den nationalen Rahmen des französischen Verfassungsrechts hinaus Wirkung entfalten.

Die Modellbildung, zu der eine rechtsvergleichende Perspektive führt, verleiht dem Juristen Unabhängigkeit gegenüber Zeit und Raum, die gemäß der idealistischen Denktradition lediglich der sinnlich erfahrbaren Welt innewohnende Elemente sind, einer Welt, die, weil sie ein geliehenes, vielschichtiges und relatives Wesen ist, stets im Werden und in permanenter Bewegung befindlich, keine echte Existenz besitzt und im Gegensatz zu den Ideen niemals ist.

Die rechtsvergleichende Perspektive nimmt zunächst einmal eine räumliche Distanz ein, insofern der geschaffene wissenschaftliche Gegenstand aus einem Konzept besteht, das auf der Grundlage einer Analyse der Vielfalt der positiven Rechtsordnungen gewonnen wird. Greift der Verfassungsrechtler auf die Begriffe "Verfassungsgericht" oder »Oberster Gerichtshof « zurück, deren Bezeichnungen auf keine bestimmte nationale Verfassung zurückgehen, so ergibt sich der von ihm geschaffene wissenschaftliche Gegenstand aus dem Vergleich unterschiedlicher Rechtsordnungen - womit sich die enge Verbundenheit von theoriegestütztem Vorgehen und rechtsvergleichender Betrachtung zeigt. Die Vergleichende Rechtswissenschaft ist nicht die Beschäftigung mit dem ausländischen Recht, sondern ein vom rechtsvergleichenden Juristen durch eine Analyse der verschiedenen nationalen Rechtsordnungen geschaffener Gegenstand. Rechtsvergleichung existiert nicht in Form positiven Rechts, sondern in Form eines durch Modellbildung geschaffenen und auf der Metaebene stattfindenden Diskurses, und dadurch ist die in anderen Rechtszweigen häufig anzutreffende Gefahr der Verworrenheit zwischen der diskursiven Ebene des positiven Rechts und der metadiskursiven Ebene der Rechtswissenschaft in der Vergleichenden Rechtswissenschaft ausgeschlossen. Im Gegensatz etwa zum französischen Recht, zum britischen Recht, zum europäischen Recht oder allgemein zu jeder positiven Rechtsordnung, die stets einer räumlichen Beschränkung unterliegen und - um die Formulierung Kelsens aufzugreifen - eine "Selbstdeutung" liefern, »die der von der Rechtserkenntnis $\mathrm{zu}$ leistenden Deutung vorgreift «, ${ }^{18}$ ist die Rechtsvergleichung aufgrund der bloßen Tatsache, dass sie nicht als positive Rechtsordnung existiert, ein reines Wissen und bei der Konstitution seines Gegenstands so souverän wie jede andere nichtrechtliche Wissenschaft, also ein reines Konstrukt des Rechtswissenschaftlers. Ihr Gegenstand, Ergebnis des dem Beruf des Wissenschaftlers innewohnenden ordnenden und systematisierenden Vorgehens, besteht aus einer Reihe von Aussagen, die für jedes nationale Recht gültig sind und eine konzeptuelle Einheit bilden, die aufgrund einer 
Analyse der empirischen Vielfalt rechtlicher Phänomene gewonnen wurde. Die Rechtsvergleichung scheint daher innerhalb der Juristengemeinschaft die freieste Herangehensweise in Bezug auf die empirische Realität, aus der sie ihren Gegenstand gewinnt, darzustellen. Bei den Juristen bildet sie den archetypischen Ausdruck eines theoriegeleiteten Gedankengangs. ${ }^{19}$

Die Modellbildung befreit den Juristen zudem von zeitlichen Zwängen. Modellbildung, die ganz allgemein jeder Jurist, der theoretisch arbeiten will, vornimmt, und der sich der rechtsvergleichende Jurist durch seinen von den Zufälligkeiten einer bestimmten Rechtsordnung losgelösten Vorgehensweise ganz selbstverständlich widmet, hat den Vorteil, den wissenschaftlichen Gegenstand des Verfassungsrechtlers vor den Unwägbarkeiten der Entwicklung des positiven Rechts und der rechtlichen Momentaufnahme zu schützen. Mehr noch als eine reine Exegese bewahrt die Konzeptualisierung den Metadiskurs des Rechtswissenschaftlers vor den Folgen einer Vergreisung. Gemäß der idealistischen Denkschule ist nämlich die Idee (Platon) bzw. das Ding an sich (Kant) unabhängig von jeder Art von Zeitlichkeit, die nach Ansicht eines weiteren Idealisten, Schopenhauer, lediglich zur »Welt als Vorstellung « zählt. ${ }^{20} \mathrm{Nach}$ Platon, dem berühmten Begründer der Akademeia, ist Zeit nichts anderes als das »bewegliche Abbild der Ewigkeit ${ }^{21}$ sodass Begriffe - die per definitionem keine empirische Dimension beinhalten - ahistorisch sind und aufgrund ihrer Allgemeingültigkeit dem Schrifttum des Juristen einen hinreichenden Schutz vor geistiger Obsoleszenz bieten. Eine zum "Standardwerk" avancierte wissenschaftliche Veröffentlichung hat umso größere Chancen, auch von künftigen Generationen Beachtung zu erhalten, je höher ihr Grad an Allgemeinheit ist, wohingegen beispielsweise eine Rechtsprechungschronik Gefahr läuft, stark von den zufälligen und bereits ihrem Wesen nach flüchtigen Gegebenheiten des Augenblicks abzuhängen und weniger Spuren für die Nachwelt zu hinterlassen. In dem Maße, in dem eine Chronik, wie bereits die Herkunft dieses Wortes nahelegt, mit dem Konzept der Zeit verwoben ist - hier derjenigen, in der die betreffende Rechtsprechung ergangen ist -, muss deren Autor eine verstärkte Anstrengung leisten, wenn er ihren wissenschaftlichen Wert über die Flüchtigkeit seiner Gegenwart hinaus sichern will; dafür muss ein solcher Autor auf eine durch die rechtsvergleichende Analyse beförderte Modellbildung zurückgreifen.

So schreibt etwa Michel Troper, dass, wer etwas anderes hervorbringen wolle als eine banale und "völlig bedeutungslose Beschreibung, die nichts weiter wäre als eine bloße Wiedergabe der Aussagen des französischen positiven Rechts [...], das positive Recht über den Verfassungsrat mit Metakonzepten wie 'Normenkontroller, >Verfassungsprozess` oder /Verfassungsgericht` erfassen [müsse], die im Verfassungstext selbst nicht aufgeführt sind $\omega^{22}$ Dem sei hinzugefügt, dass diese Metakonzepte für alle nationalen Rechtsordnungen gültig sind, in denen verfassungsgerichtliche Normenkontrollverfahren vorgesehen sind. Eine Auseinandersetzung mit dem positiven Recht jenseits der nationalen Grenzen fördert und erleichtert eine Modellbildung, die dem vom Juristen geschaffenen wissenschaftlichen Gegenstand einen festen Schutz gegen das Werk der Zeit bietet.

Angesichts der Wende hin zum Rechtsprechungskommentar und der mit dieser Wende verbundenen Gefahr, lediglich eine Momentaufnahme des Rechts abzubilden, kommt der Verfassungsrechtler somit nicht umhin, eine theoretische und für die Rechtsvergleichung offene Vorgehensweise zu wählen, um einen stärkeren Grad an Allgemeinheit zu erreichen und den wissenschaftlichen Charakter seines Diskurses zu 
bewahren. Gleichzeitig darf er nicht verkennen, dass - in jeder Disziplin - ein Gelehrter den Gegenstand seiner Wissenschaft konstruiert, indem er - seit der modernen epistemologischen Revolution im 18. Jahrhundert - sich methodisch des Instruments des Erkennens von Kausalitätszusammenhängen bedient. Will der Verfassungsrechtler den von Gaston Bachelard formulierten konstruktivistischen Anforderungen genügen, darf er auf dieses Instrument nicht verzichten, selbst wenn sein Interesse eher den verfassungsprozessrechtlichen Aspekten des Verfassungsrechts gelten sollte.

\section{Kausalität als idealer Modus der Konstruktion des Gegenstands der Verfassungswissenschaft}

Um seinen wissenschaftlichen Gegenstand zu konstruieren, erzeugt der Rechtsgelehrte theoretische Aussagen, die in der Rechtswissenschaft den wissenschaftlichen Gesetzen in allen anderen Wissenschaften entsprechen: Sie dienen der Gewinnung von Erkenntnissen, womit sie sich von den normativen Gesetzen unterscheiden, die von den rechtssetzenden Organen in deren Funktion als Regulierer menschlichen Verhaltens erlassen werden. Die Unterscheidung zwischen wissenschaftlichen Gesetzen und normativen Gesetzen darf jedoch jene als ausschließlich beschreibende Aussagen gegenüber diesen nicht in den Hintergrund treten lassen. Wissenschaftliche Gesetze sind durchaus Gesetze, und gemäß der treffenden Formulierung von Paul Amselek erweisen sie sich als "geistige Werkzeuge, die die Wahrscheinlichkeit für den Eintritt bestimmter Dinge messen «. ${ }^{23}$ Sie sind, fügt er hinzu, "nach folgendem Schema formuliert: Unter diesen oder jenen Bedingungen wird sich, kann sich nicht oder kann sich dieses oder jenes Phänomen ereignen - bzw., in Wahrscheinlichkeiten ausgedrückt, besteht eine bestimmte Wahrscheinlichkeit für ihr Eintreten«. ${ }^{24}$ Diese Strukturierung des Gedankengangs hat einen Namen: Kausalität, als ein wesentliches Merkmal theoretischer Aussagen, ebenso wie deren Allgemeinheit. Wissenschaftliche Gesetze sind sehr wohl Gesetze, und ihre Besonderheit liegt darin, auf Kausalzusammenhängen zu beruhen.

Aufgrund der normativen Besonderheit des Materials, mit dem sich die Rechtswissenschaft beschäftigt, erachtete Kelsen es als unmöglich für Juristen, auf Kausalzusammenhängen beruhende Gesetzmäßigkeiten herauszuarbeiten. Rechtliche Normen seien ideelle Einheiten, denen ein anderes Strukturprinzip zugrunde liege: die Zurechnung, ein wesentliches Merkmal der Welt des Rechts. Kelsen zufolge hat diese ontologische Eigenschaft der Normen Auswirkungen auf die Rechtswissenschaft, die sich zwangsläufig von den Kausalwissenschaften (Naturwissenschaften und nichtnormative Sozialwissenschaften), »die auf die kausalgesetzliche Erkenntnis tatsächlicher Vorgänge« abzielten, abgrenzt. ${ }^{25}$ Mit anderen Worten, für Kelsen hing die Epistemologie in Bezug auf das Recht von der ontologischen Struktur der Welt des Rechts ab.

Diese Ansicht erscheint uns gefährlich vereinfachend, denn sie kann eigentlich nur dazu führen, die Rechtswissenschaft als bloßes semantisches Abbilden der Rechtsnormen zu betrachten und diese Wissenschaft zu einer Aussätzigen gegenüber allen anderen wissenschaftlichen Disziplinen zu degradieren. Rechtswissenschaft wäre dann die einzige Disziplin, die von der ontologischen Struktur der von ihr untersuchten Welt abhinge. Tatsächlich hat diese aus ideellen Einheiten bestehende und vom Grundsatz der Zurechnung geleitete Welt für die unglücklicherweise von der 
Kelsen'schen Überschätzung der Besonderheit ihrer Disziplin beeinflussten Juristen lange ein furchtbares Erkenntnishindernis dargestellt (A). Erst im Zuge der Wende hin zum Rechtsprechungskommentar und der wachsenden Bedeutung prozessrechtlicher Fragen innerhalb seines eigenen Fachbereichs hat der Verfassungsrechtler die ganze Wucht dieses durch die ideelle Dimension der Normen begründeten Hindernisses zu spüren bekommen. Wie jeder andere Jurist auch hat er, wenn er dieses Hindernis umgehen will, keine andere Wahl als den Gegenstand seiner Wissenschaft zu einem Gegenstand empirischer Untersuchungen zu erklären, der mittels Kausalitätszusammenhängen betrachtet werden kann, indem das Interesse des Wissenschaftlers über die semantische Ebene der Richtersprüche auch der pragmatischen Dimension dieses Diskurses gilt (B).

\section{A. Ein Erkenntnishindernis: die ideelle Dimension der Normen}

28 In den empirischen Wissenschaften, welche die Naturwissenschaften und die nichtnormativen Gesellschaftswissenschaften (d.h. sämtliche Sozialwissenschaften mit Ausnahme der Rechtswissenschaft) einschließen, ist das Kausalitätsprinzip das intellektuelle Standardwerkzeug des Wissenschaftlers zum Begreifen der Welt. Es verbürgt die Wissenschaftlichkeit des akademischen Diskurses, denn da weder der Natur noch der Gesellschaft inhärent, sondern ausschließlich dem Geist des Forschers entsprungen, bezeugt es die konstruktive Dimension der wissenschaftlichen Vorgehensweise.

29 Es sei daran erinnert, dass Kausalität kein ontologisches Merkmal der physischen Welt ist, der Grundsatz der Zurechnung dagegen ein ontologisches Merkmal der spezifischen Welt der Normen. Wie vom skeptischen Empiriker David Hume treffend dargelegt wurde, ist die Regelmäßigkeit, die von den Kausalitätsgesetzen aufgezeigt wird, wenn diese die innerweltlichen Sachverhalte nach dem Ursache-Wirkung-Prinzip darstellen, ausschließlich postuliert und erschließt sich nicht von selbst. ${ }^{26}$ Zwischen der beobachteten Regelmäßigkeit eines besonderen Phänomens und der Formulierung eines Gesetzes, das dieses rational erfasst und eine allgemeingültige Aussage aufstellt, liegt ein qualitativer Sprung, der nichts Rationales an sich hat und lediglich eine Überzeugung ausdrückt, die Überzeugung nämlich, dass das beobachtete Phänomen sich unendliche Male wieder ereignen wird; es ist eine Wette auf die Zukunft, gewissermaßen eine Handlung, die mehr dem Willen als dem Wissen geschuldet ist insofern ist der Begriff des "Gesetzes" diesbezüglich gerechtfertigt. Es handelt sich in der Tat um ein geistiges Konstrukt, das der Wissenschaftler nicht in die Welt projiziert, das der Laie jedoch als deckungsgleich mit der Welt verwechselt. Paul Amselek hat diesen Aspekt gut erkannt, wenn er eine hartnäckige und in unserem Denken weitverbreitete transzendentale Illusion anprangert, die er als Form der "Verweltlichung" des Denkens und der Auslegung der Welt am Maßstab des menschlichen Wesens ansieht. Diese Neigung besteht seiner Ansicht nach darin, »das, was der Geist des handelnden Wissenschaftlers [tut], auf die Welt, die Gegenstand seiner Analyse ist, zu projizieren und zu glauben, die Regelmäßigkeiten, die Gesetze, die Vorhersehbarkeit, die Ordnung seien Merkmale, Wesenszüge besagter Welt, Bestandteile und Modalitäten von deren ontologischer Struktur, welche die besagte Welt der Betrachtung durch den Wissenschaftler anbiete ${ }^{27}{ }^{27}$ wohingegen richtigerweise diese Gesetze nur ein Konstrukt des Geistes und nicht Gegebenheiten der analysierten Welt seien. 
30 Der vom Laiendenken nur unzureichend erfasste konstruierte Charakter von Kausalitätszusammenhängen ist im Übrigen der Garant für die Allgemeingültigkeit wissenschaftlicher Gesetze, insofern die Welt für sich genommen nur aus Vielfalt und Kontingenz besteht. ${ }^{28}$ Konstruiert und ohne eigene weltliche Existenz, ist die Allgemeingültigkeit das Ergebnis eines induktiven Denkprozesses, der darin besteht, auf der Grundlage regelgerechter, aber kontingenter Beobachtungen allgemeine Gesetze abzuleiten. Ein solches Vorgehen ist gelungen, wenn die Regelmäßigkeit eines Vorgangs Ausdruck einer systematischen Verkettung von Ursache und Wirkung ist, der sich auch bei ähnlich gelagerten Phänomenen unendliche Male wiederholen kann. Kausalität und Allgemeinheit sind daher miteinander verbunden. Diese Verschränkung belegt den konstruierten Charakter wissenschaftlicher Gesetze, deren Zweck darin liegt, ein abstraktes Universum zu erschaffen, das zu der sinnlich erfahrbaren Welt hinzutritt und diese vorstellungsgemäß und diskursiv in Kategorien ordnet. Diese Gesetze stellen die Vorstellung dar, die die sinnlich erfahrbare Welt repräsentiert, der die Kausalität fremd ist - diese existiert ausschließlich im Denken des Gelehrten. Die Diskrepanz zwischen dem wissenschaftlichen Diskurs und den Dingen der Welt, die durch diesen Diskurs rational erfasst werden, hängt mit diesem Fehlen von Kausalität zusammen. Daraus ergeben sich der konstruierte Charakter des wissenschaftlichen Gegenstands und die Wissenschaftlichkeit des Diskurses. Kausalität, Allgemeinheit, Künstlichkeit des Gegenstandes stehen in engem Zusammenhang und sichern so die Wissenschaftlichkeit des Diskurses.

31 Wie verhält es sich in Bezug auf die Welt des positiven Rechts, die für die Rechtswissenschaft dem entspricht, was die sinnlich erfahrbare Welt für die empirischen Wissenschaften ist? Auch hier gilt, dass die Kausalität nicht von dieser Welt ist, wodurch der Gedanke naheliegend wäre, dass ein von der Rechtswissenschaft formulierter Satz, um mit der von ihr analysierten Welt diejenige kritische Distanz zu wahren, die ihr eine eigenständige Erschaffung ihres wissenschaftlichen Gegenstandes erlaubt, auf einer ebenso weißen Leinwand entstehen könnte wie sie beim Formulieren jedes wissenschaftlichen Gesetzes gegeben ist. Dem ist jedoch nicht so. Ein solcher Abstand ist für Juristen nur schwer zu erreichen. Zwar ist Kausalität der Welt der Normen offensichtlich fremd, doch zeichnet sich diese vom Rechtswissenschaftler betrachtete besondere Welt durch eine andere Art von Zusammenhang aus: durch den Grundsatz der Zurechnung. ${ }^{29}$ Während Kausalität in der erforschten Welt lediglich ein geistiges Konstrukt ist, ist Zurechnung selbst eine Gegebenheit der Welt des Rechts. Infolgedessen behindert die Tatsache, dass innerhalb dieser Welt des Rechts die Normen durch Zurechnung miteinander verbunden sind, den Juristen bei seinem Bemühen, wissenschaftliche Eigenständigkeit $\mathrm{zu}$ erreichen, denn diese VorBestimmtheit kann ohne weiteres den Inhalt des wissenschaftlichen Diskurses beeinflussen. Schließlich ist die Norm, eine ideelle Einheit, der Bedeutungsinhalt einer Aussage, die, wenn gewisse Bedingungen gegeben sind, normative Folgen einem diese Folgen auslösenden Sachverhalt zurechnet. Sie ist der Bedeutungsinhalt einer Aussage über das, was sein soll, und erscheint damit als Bedeutungsinhalt einer Idealität und nicht einer Realität. Folglich muss der Rechtssatz - die Aussage, mit der ein Rechtswissenschaftler diese Idealität beschreibt - zwangsläufig den Zurechnungszusammenhang, aus dem diese Idealität besteht, abbilden, und weist vom strukturellen Aspekt her keine Eigenständigkeit gegenüber der von ihm erfassten Norm auf. Angesichts dieser nicht zur sinnlich erfahrbaren Welt gehörenden ideellen Einheit ist für den Juristen die Versuchung groß, gewissermaßen aus einer Art 
intellektueller Bequemlichkeit heraus, diesen Zurechnungszusammenhang im Rahmen der Formulierung dessen lediglich zu wiederholen, was Kelsen ein beschreibendes Sollen nennt, d. h. einen Satz zu formulieren, dessen Bedeutungsgehalt die Nachbildung der Normaussage ist, was vom wissenschaftlichen Standpunkt aus betrachtet keinen Erkenntnisgewinn bietet. Denn der Inhalt der Norm als ideeller Einheit, selbst wenn er von einem Richter in seinem Urteilsspruch herausgearbeitet wird, ist nichts weiter als der Gedanke, dass »ein bestimmtes Verhalten erfolgen soll«. Somit weist der diesen Gedanken ausdrückende und von Kelsen als »beschreibendes Sollen« bezeichnete Rechtssatz keinen anderen Gehalt auf als die Norm selbst, d.h. den Gedanken, dass »eben dieses (von der Norm vorgeschriebene) Verhalten erfolgen soll«. Unter dem Aspekt der Erkenntnis gesehen, ist der Rechtssatz eine entbehrliche Aussage, da er keinen eigenen Inhalt aufweist. Er ist transparent, gewissermaßen das Spiegelbild der Norm. Die bereits in der Norm angelegte Zurechnung äußert sich in identischer Weise im Rechtssatz. Während Kausalitätszusammenhänge das geistige Werkzeug des Gelehrten in den empirischen Wissenschaften darstellen und ausschließlich dessen Geist entspringen, existiert die Zurechnung bereits, nämlich in den Normen - sie gehören zu deren ureigenen Merkmalen. Aufgrund dieser ontologischen VorBestimmtheit beeinflusst die Zurechnung die Sätze der Rechtswissenschaft, die ihrerseits nur ein blasses Abbild von ihr bilden. Im Gegensatz $\mathrm{zu}$ den wissenschaftstheoretisch eigenständigen empirischen Wissenschaften ist die Rechtswissenschaft dazu verurteilt, eine fade Nachbildung des positiven Rechts zu sein, wenn der Jurist sich auf eine bloße Normexegese beschränkt.

Um dieses erkenntnistheoretische Dilemma zu überwinden und sich von der Formulierung der Norm abzugrenzen, deren reine Exegese dem Juristen zu Recht als unproduktiv erscheint, besteht der von diesem häufig gewählte Weg darin, mögliche Interpretationen der Norm herauszuarbeiten. Will er die wissenschaftlich reizlose und bloß beschreibende Textexegese vermeiden, kann der Jurist die genau entgegengesetzte Vorgehensweise wählen und vorschreiben, was sein sollte, was dazu führt, dass er die Grenzen des wertmäßigen Neutralitätsgebots der Rechtswissenschaft überschreitet. Denn auslegen erfordert auch bewerten - mit anderen Worten eine Festlegung, eine Wahl, die sich per Definition der Objektivität entzieht. Diesen - nichtwissenschaftlichen - Weg beschreitet die Dogmatik. Dazu gehören eine ganze Reihe hermeneutischer Verfahren, beispielsweise das des Rechtsanwalts oder des Rechtslehrers, der eine Rechtsberatung erteilt, oder auch des Rechtsgelehrten, der eine rein subjektive Auslegung einer gesetzlichen (oder verfassungsrechtlichen) Bestimmung vornimmt, die noch nicht Gegenstand einer sogenannten authentischen Interpretation durch den Richter war.

Gemäß den voluntaristischen Theorien der Interpretation kennt niemand die geltende Norm, bevor nicht der Richter die sogenannte authentische Interpretation der betreffenden Bestimmung vornimmt. Die Debatten in der Rechtsliteratur kreisen somit um die Potenzialität mehrerer anwendbarer Normen ${ }^{30}$ Besteht die Tätigkeit der Rechtswissenschaft im Kelsen'schen Sinne darin, eine abschließende Aufzählung dieser verschiedenen möglichen Normbedeutungen vorzunehmen, indem sie eine Reihe von Sollen-Zuständen beschreibt und das umsetzt, was bei Kelsen als wissenschaftliche Interpretation bezeichnet wird, ${ }^{31}$ so tritt sie hingegen zugunsten der Dogmatik in den Hintergrund, wenn der Jurist unter den zahlreichen möglichen Auslegungen eine Wahl trifft und begründet, welche ihm am angebrachtesten scheint. Er tritt damit als Vertreter einer Lehrmeinung auf. Beispielhaft sei hier die den Entscheidungen des 
Verfassungsrates ${ }^{32}$ und des Kassationsgerichtshofes ${ }^{33}$ vorausgehende Debatte um die alte Fassung von Artikel 68 der französischen Verfassung und die Frage der strafrechtlichen Haftung des Staatspräsidenten genannt. Dabei handelt es sich um eine Debatte in der Rechtslehre, deren jeweilige Protagonisten Festlegungen vornahmen, um semantische Mehrdeutigkeiten aufzulösen, indem sie verschiedene Lesarten der Vorschrift von Artikel 68 (alte Fassung) der Verfassung anboten. ${ }^{34}$ Hier zeigt sich der Beitrag der Rechtslehre (der Dogmatik im Allgemeinen): Sie leistet die gleiche Arbeit wie der Richter, allerdings ohne dessen Befugnis zu besitzen, eine authentische Interpretation vorzunehmen. Anders gesagt, steuert sie im Vorfeld die hermeneutische Tätigkeit des Richters und erhebt sich zu einer indirekten Rechtsquelle. Erst wenn der Rechtsprechungsprozess abgeschlossen ist und der Richter die authentische Interpretation vorgenommen hat, nimmt der Jurist wieder seine beschreibende Position ein. Indem er als Rechtsprechungskommentator die geltende Norm beschreibt, wobei er erneut der Gefahr ausgesetzt ist, beschreibende Soll-Zustände ohne wissenschaftliche Relevanz $\mathrm{zu}$ formulieren, beschreibt er, was sein soll, auf der Grundlage dessen, was der Richter aus der anwendbaren Vorschrift herausgelesen hat, und zwar mittels Sätzen der Art »der Richter hat entschieden, dass«. Erneut sieht sich der Jurist konfrontiert mit dem Dilemma, das der Mangel an Eigenständigkeit des rechtswissenschaftlichen Metadiskurses gegenüber der Normaussage bewirkt: der Jurist hat sein epistemologisches Problem nicht gelöst, sondern in unproduktiver Weise lediglich in die Phase der Rechtsfindung durch den Richter verschoben.

Es zeigt sich, dass in einer Welt, die aus Aussagen besteht, deren Sinn das betrifft, was sein soll, der Jurist gefangen ist in den engen Grenzen einer hermeneutischen Tätigkeit, die ihn dazu bringt, entweder diese Aussagen in beschreibender Weise und ohne wissenschaftliche Relevanz wiederzugeben oder sich von ihnen zu befreien, jedoch um den Preis einer präskriptiven Vorgehensweise ohne Wissenschaftlichkeit. Unter solchen Umständen besteht der Ausweg für einen Rechtsprechungskommentator, der eine wissenschaftliche Leistung erbringen will, die weder deskriptiv noch präskriptiv ist, darin, sein Interesse vom Aspekt des Sinns und des normativen Charakters der Aussagen des Gerichts wegzulenken und auf das zu richten, was das Gericht tut, wenn es diese Aussagen formuliert. Ein Kommentator von Urteilen, der diesen Weg geht, gibt dem pragmatischen Aspekt des richterlichen Diskurses den Vorzug und schafft sich selbst einen Gegenstand empirischer Untersuchungen, den er unter Kausalitätszusammenhängen betrachten kann. ${ }^{35}$

\section{B. Eine unverzichtbare Hilfsquelle: die pragmatische Dimension der Sprache des Richters}

Konfrontiert mit der erkenntnistheoretischen Sackgasse einer ausschließlichen Befassung mit dem semantischen Aspekt des Urteilsspruchs, beweisen einige Urteilskommentatoren Hellsichtigkeit. Dies belegen deren Rechtsprechungschroniken, in denen sie dem Leser offenbaren, was nicht unmittelbar aus dem Urteilsspruch des Richters ersichtlich ist. Ergiebige Beispiele zeigen sich in vielen Fällen, wie insbesondere in der Anfangszeit nach der Einführung des neuen prozessrechtlichen Mittels der vorrangigen Frage zur Verfassungsmäßigkeit (QPC): So erkannten manche Kommentatoren in Beschlüssen des Staatsrats oder des Kassationsgerichtshofs, mit denen eine vorrangige Frage zur Verfassungsmäßigkeit wegen Nichterfüllung der Voraussetzung der Ernsthaftigkeit der Frage verworfen wurde, die Durchführung einer 
verkappten Normenkontrolle, indem diese obersten Gerichte das gerügte Gesetz auf solch eine Weise auslegten, dass es als verfassungskonform gelten konnte. ${ }^{36}$ Indem er seine Aufmerksamkeit der versteckten Seite des Richterspruchs widmet (nämlich, dass darin eine diffuse Normenkontrolle zum Ausdruck kommt), seziert der Urteilskommentator die Wirklichkeit in einer Weise, die dem konstruktivistischen Ideal des theoretischen Gedankengangs Genüge tut und der Abhandlung des Kommentators einen hinreichend wissenschaftlichen Charakter sichert. Daran ändert sich auch nichts, wenn der Kommentator sich eine kritische Würdigung der Vorgehensweise des Gerichts erlaubt und, in einem solchen Fall, eine Lehrmeinung vertritt, die ihn nur in seiner Eigenschaft als Bürger bindet. Die wissenschaftliche Relevanz seines Kommentars liegt dann weniger darin, dass er diese subjektive Bewertung vornimmt, als in seiner Fähigkeit zu enthüllen, wie der Richter vorgegangen ist.

Indem er auffordert, hinter der Prüfung des Zulässigkeitskriteriums der Ernsthaftigkeit einer vorrangigen Frage zur Verfassungsmäßigkeit die tatsächliche Durchführung einer (jedoch nicht offen als solche bezeichneten) konkreten Normenkontrolle zu sehen, erkennt der Urteilskommentator das Göttliche in einer Sache und schafft durch diese theoretische Vorgehensweise die für die Wissenschaftlichkeit seines Metadiskurses unerlässliche Distanz zwischen diesem Diskurs und der vom Richter ausgesprochenen Normaussage. Hierin liegt der schmale und schwierige Pfad, den sich die Rechtswissenschaft bahnen muss zwischen auf der einen Seite dem bloß empirischen und unschöpferischen Ergebnis, zu dem eine beschreibende Darlegung von SollZuständen führt, und auf der anderen Seite der normformulierenden Vorgehensweise von Lehrmeinungen. Hier ist der Kant'sche Weg des Kritizismus deutlich zu erkennen, der darin besteht, weder zu beschreiben noch vorzuschreiben, sondern zu erschaffen. Wissenschaftliche Erkenntnis bietet einen kognitiven Mehrwert, nämlich eine Vorgehensweise, die etwas anderes "ausspricht« als das, was ein Beobachter ohnehin unmittelbar erkennen kann, wobei hervorzuheben ist, dass besagter Mehrwert, der eher von der Konstruktion als vom Wissen abhängt, in einem Denkmodell besteht, das auf der Grundlage einer kritischen Beobachtung der Sachlage jenseits einer passiven Lektüre von Aussagen herausgearbeitet wird. ${ }^{37}$

Der paradoxe Charakter dieses Vorgehens beruht darin, dass der Kommentator von Urteilen, wenn er durch die Einnahme einer theoretischen Perspektive, die ihn von einer fruchtlosen empiristischen Lesart der Urteilssprüche des Richters entfernt, das Göttliche in einer Sache erkennen will, auf der Grundlage dessen, was er von dieser Lektüre des Urteils mitnimmt, einen Gegenstand empirischer Untersuchungen entwickeln muss. Dieser Gegenstand, erkennbar lediglich im pragmatischen Aspekt des Urteilsspruchs, ist »das-was-der-Richter-tut-wenn-er-Recht-spricht«. Indem er seinen Gegenstand zu einem empirisch fassbaren Gegenstand erhebt, lässt der Jurist die rein empiristische Haltung desjenigen hinter sich, der seine Aufgabe auf eine bloße Wiedergabe des Inhalts der Gerichtsentscheidung beschränkt. Durch Dekonstruktion des Urteils, hinter dessen semantischen Schleier er schaut, offenbart sich dem Juristen die verborgene Seite der Dinge. Um zur Idee durchzudringen und das Wesen der Welt zu erfassen, erschafft sich der Jurist paradoxerweise einen empirischen Gegenstand. Daraus ergeben sich für ihn drei Vorteile, welche die Wissenschaftlichkeit seiner Vorgehensweise verbürgen. 
richterliche Tun, dessen Entwicklung jenseits des richterlichen Diskurses der Jurist aufzeigt, ist kein dem Wissenschaftler vorgegebener Gegenstand, sondern einer, den dieser sich selbst erschafft. Es ist ein im Bachelard'schen Sinne konstruierter Gegenstand, Ergebnis einer geistigen Erfassung und Ordnung des dem Urteilskommentator gegebenen Rohmaterials. Bei diesem Rohmaterial handelt es sich um nichts anderes als um die Unordnung der vom Richter öffentlich formulierten Normaussagen, deren bloßes Lesen nicht zur Konstitution des wissenschaftlichen Gegenstands führen kann. Das, was der Wissenschaftler entwickelt, wenn er die pragmatische Dimension des Richterspruchs beleuchtet, ist jenseits des Gesagten, liegt in dessen illokutionärer Dimension. ${ }^{38}$ Die Konstitution des empirischen Gegenstands steht am Ende eines höchst idealistischen Vorgehens. Dieser Gegenstand wird nicht unmittelbar durch die Lektüre der Entscheidungsgründe erkannt, die für den Kommentator nur die oberflächliche Schale des Urteils darstellen, sondern mittelbar durch den Verstand, der ihm dank des theoretischen Vorgehens ermöglicht, das Göttliche in einer Sache zu sehen, und ihm Aufschluss darüber gibt, was der Richter tatsächlich tut, wenn er Recht spricht. Wie es die Besonderheit der konstruktivistischen und theoretischen Vorgehensweise fordert, muss der Kommentator von Urteilen die empirische Realität dessen, was der Richter tut, durch die Augen der Seele betrachten.

Der zweite epistemologische Vorteil für den Urteilskommentator, wenn er seine Aufmerksamkeit auf den pragmatischen Aspekt der verfassungsgerichtlichen Urteilssprüche richtet, liegt in den intrinsischen Eigenschaften des empirischen Gegenstands. Von dem Moment an, an dem der Gegenstand empirisch untersucht werden kann, ist es ebenfalls möglich, ihn unter Kausalitätsgesichtspunkten zu behandeln. Ohne jegliche ideelle Dimension und vom Ballast der Zurechnung befreit, wird er zu einem Gegenstand ontologisch gleicher Art wie in den empirischen Wissenschaften (Naturwissenschaften und nicht-normative Sozialwissenschaften) und bietet dem Urteilskommentator - wie jeder derart beschaffene Gegenstand wissenschaftliche Eigenständigkeit, die der Kommentator für sich nutzt, indem er Erklärungsmuster kausaler Art in Bezug auf die Gerichtentscheidung herausarbeitet, die sich dafür interessieren, wie die Entscheidung erarbeitet wurde, unter welchen Umständen sie entstanden ist oder auch welchen rechtlichen Zwängen der Richter bei seiner Entscheidungsfindung unterliegt. ${ }^{39}$ An dieser Stelle sei das bereits genannte Beispiel wieder aufgegriffen: das Verhältnis von Staatsrat und Kassationsgerichtshof zum Verfassungsrat, wenn es um die Prüfung vorrangiger Fragen zur Verfassungsmäßigkeit geht, und das entweder als Konkurrenzsituation (aus Sicht pessimistischer Kommentatoren) oder als Kooperationsverhältnis (aus Sicht optimistischer Kommentatoren) gesehen wird. Dieses Verhältnis kann vom Juristen als eine Gegebenheit soziologischer Art gesehen werden, die ihm die Möglichkeit eröffnet, näher zu beleuchten, was der Richter eigentlich tut, wenn er entscheidet, dass eine gegen eine bestimmte gesetzliche Vorschrift gerichtete vorrangige Frage zur Verfassungsmäßigkeit der Ernsthaftigkeit entbehrt: in dem Fall erhebt sich der Richter nämlich zum Verfassungsrichter. Hier bietet sich dem Juristen die günstige Gelegenheit, für die wissenschaftliche Untersuchung verfassungsgerichtlicher Entscheidungen auf eine Wissenschaft zurückzugreifen, die sich mit den Verhaltensweisen der Richter beschäftigt und zu den nicht-normativen soziologischen Disziplinen gehört, wie bspw. die Politikwissenschaft. Dadurch, dass er aus der Rechtswissenschaft eine empirische Wissenschaft macht anstelle einer normativen, 
kann der Jurist seinen wissenschaftlichen Gegenstand erschaffen, was im Übrigen der methodischen Anforderung entspricht, die bereits von Léon Duguit formuliert wurde, der zu Recht forderte, die rechtswissenschaftlichen Fakultäten müssten die Bezeichnung »Rechts- und Sozialwissenschaftliche Fakultäten« tragen. Die Einbettung der Rechtswissenschaften in die nicht-normativen Sozialwissenschaften stellt sich als conditio sine qua non dar, um dem Juristen die wissenschaftliche Eigenständigkeit zu sichern, wohingegen umgekehrt die ausschließliche Berücksichtigung dessen, was die Besonderheit der Normativität ausmacht, nämlich deren ideelle Dimension, für den Juristen ein ernstes Erkenntnishindernis darstellt: Die vom Juristen für die Konstitution seines Gegenstandes benötigte Autonomie erfordert, logischer- ebenso wie paradoxerweise, eine gleichgültige Haltung in Bezug auf die ontologische Eigenständigkeit des Rechts und die ausdrückliche Forderung nach epistemologischer Interdisziplinarität zwischen Juristen und Nichtjuristen. Aus diesem Grund würde der bei manchen Juristen latent vorhandene und mit der Besonderheit der Welt des Rechts begründete Wunsch nach einer Eingliederung der rechtswissenschaftlichen Fakultäten in die Organisationsstruktur des Justizministeriums endgültig die Totenglocke für die Rechtswissenschaft läuten. Das Recht wäre dann nur noch für diejenigen von Belang, die es anwenden, und nicht mehr für diejenigen, die es erforschen. Gefangen im Käfig einer Institution, die ausschließlich eine technische Ausbildung liefern würde, richteten die Juristen ihren Fokus nur noch auf den semantischen Aspekt der Gerichtsurteile und sähen daher nur noch Schatten.

Es gibt für den Urteilskommentator, der gewillt ist, seinen wissenschaftlichen Gegenstand jenseits der Bedeutungsinhalte der Sprache der Gerichte zu konstruieren, noch einen dritten Vorteil. Indem er sein Interesse auf das richtet, was der Richter in Wirklichkeit tut, wenn er sein Urteil formuliert, zeigt der Kommentator, dass er dem Weisen gleich verstanden hat, dass er stets nur eine oberflächliche Erkenntnis der Welt des Rechts erreichen wird, wenn er sich nicht aus dem Gefängnis der Platon'schen Höhle befreit, in das ihn die reine Textexegese sperrt. Jede theoretische Herangehensweise, auch wenn diese Bezeichnung nicht ausdrücklich in Anspruch genommen wird, reiht sich zwangsläufig in eine idealistische Perspektive auf die Erkenntnis ein: Aufdecken, welche geistige Wahrheit sich hinter den Erscheinungen verborgen hält, enthüllen, was der Richter jenseits seines Urteilsspruchs tut, das Göttliche in den Dingen erblicken, bedeutet, zur Idee vorzudringen, die weder der Richter noch der Rechtspraktiker sehen. Die berühmte sogenannte realistische Lehre von der Auslegung des Rechts, die bei Verfassungsrechtlern, die sich für verfassungsprozessrechtliche Fragen interessieren, großen Anklang gefunden hat, liefert dafür ein anschauliches Beispiel. Ihre Verfechter betrachten sich aufgrund ihres gewählten wissenschaftlichen Gegenstands als Realisten in dem Sinne, dass sie jenseits des offiziellen Urteilsspruchs des Richters, der vorgibt, sich auf die Anwendung bestehender Regeln zu beschränken, zu erblicken glauben, was der Richter realiter tut, und durch diese Erkenntnis entdecken, dass der Richter durch die Auslegung der Gesetzestexte Recht setzt. ${ }^{40}$ Realisten aufgrund ihres Interesses für die pragmatische Dimension des Urteilsspruchs, sind diese Juristen in ihrer epistemologischen Haltung Idealisten. Indem er die wahre Tätigkeit des Richters jenseits von dessen Worten durchdringt und sich für den pragmatischen Aspekt des richterlichen Diskurses interessiert, ohne sich von der Semantik dieses Diskurses täuschen zu lassen, gelingt es dem den Lehren dieser Theorie gegenüber aufgeschlossenen Urteilskommentator dank dieses Enthüllungswerkzeugs, die Idee hinter den sichtbaren Dingen zu entdecken. Er sieht, wie der Richter Recht setzt, 
obgleich dieser behauptet, er beschränke sich auf dessen Interpretation, und wird dadurch zu einem Nachfolger des Kopernikus, der die Kühnheit besaß, entgegen der von der himmlischen Bahn der Sonne entstandenen Illusion das Gegenteil des für alle Sichtbaren zu postulieren, und die geozentrischen Doktrin durch die heliozentrische These ersetzte, um den Menschen zu helfen, die Wirklichkeit der Welt zu begreifen. Wer einen theoretischen Standpunkt einnimmt, betreibt immer auch eine Form von epistemologischem Idealismus.

Die Verfassungsrechtslehre, sei sie nun wohlwollend oder kritisch gegenüber der Rechtsprechung des Verfassungsrats, hat hinreichende Belege einer solchen methodischen Hellsichtigkeit gegeben, sodass es entgegen der von manchen Autoren in der jüngeren Vergangenheit geäußerten Skepsis ${ }^{41}$ erlaubt sein muss, Genugtuung darüber auszudrücken, dass diese Verfassungsrechtslehre nicht in die Falle des formalen Legalitätsdenkens getappt ist. Dies belegt ein Blick in gegenwärtige Publikationen sowie die sich daraus ergebende Feststellung, welch hohe Resonanz seitens der Rechtslehre die Entscheidung des Verfassungsrats aus dem Jahr 2010 erfahren hat, »die Verfassungsmäßigkeit der tatsächlichen Tragweite eines Gesetzes aufgrund von dessen Auslegung durch die ständige Rechtsprechung « ${ }^{42} \mathrm{zu}$ prüfen. Manche Kommentatoren haben darin scharfsinnigerweise eine Anerkennung rechtssetzender Befugnisse der obersten Gerichte der Verwaltungsgerichtsbarkeit und der ordentlichen Gerichtsbarkeit durch den Verfassungsrat gesehen, da der Rat ihrer Ansicht nach mit dieser Rechtsprechung auch die voluntaristische Theorie der Rechtsauslegung bestätigt habe, wonach ein verabschiedetes Gesetz noch keine Norm sei, solange es noch nicht durch die Rechtsprechung ausgelegt worden sei. Daraus schließen sie: »Der Verfassungsprozess greift theoretische Überlegungen auf und bietet sich diesen an! ${ }^{43}$ Ein entsprechendes Echo seitens der Rechtslehre erntete der entgegengesetzte Standpunkt des Kassationsgerichtshofes, als er sich zunächst weigerte (bevor er dann später seine Linie doch änderte), dem Verfassungsrat eine vorrangige Frage zur Verfassungsmäßigkeit vorzulegen, die das sogenannte lebendige Recht betraf. ${ }^{44}$ Indem er seine eigene Rechtsprechung vor einer Verfassungsmäßigkeitskontrolle zu bewahren suchte, zog der Kassationsgerichtshof stillschweigend eine Trennlinie zwischen Rechtsprechung und gesetzlicher Bestimmung, womit er nolens volens seine eigene etablierte Rechtsprechungsdoktrin in Frage stellte, gemäß der die Auslegungen des Gesetzes durch den Kassationsgerichtshof und das ausgelegte Gesetz eine Einheit bilden. Diese seitens der Rechtslehre scharfsichtige Analyse, die das Licht hinter dem Schatten der sich bewegenden Richter ausmachte, stammt von Nicolas Molfessis, nach dessen Ansicht die Aussage des Kassationsgerichtshofes auf "nicht mehr und nicht weniger" hinauslaufe »als der Rechtsprechung ein offizielles Dasein zu verleihen, die in einigen Entscheidungen ausdrücklich hervorgehoben wird «. ${ }^{45}$ Wenn die Richter nicht immer sehen, was sie tun, wenn sie Recht sprechen, erwartet die Rechtswissenschaft von ihren begabten Handwerkern, dass sie es sehen und sehen lassen. Auf sie zählt die Rechtswissenschaft, um die heutzutage im Verfassungsrecht erlebte Wende hin zum Rechtsprechungskommentar zu durchleben, ohne dabei ihre Identität einzubüßen.

Auf der soliden Grundlage dieser theoretischen Antworten und seines akademischen Geistes wird der heute von der kulturellen Revolution hin zum Rechtsprechungskommentar erfasste Verfassungsrechtler die wissenschaftliche Dimension seines Faches nur dann bewahren können, wenn es ihm gelingt, auf die beschriebene Art die Wirklichkeit der Welt des Rechts hinter den vom Rhythmus der zu 
entscheidenden Fälle geprägten Bewegungen der Richter in deren Platon'scher Höhle, dem Gerichtsaal, herauszulesen. Die zeitgenössische Verfassungslehre bietet in dieser Hinsicht Anhaltspunkte, die eher zuversichtlich stimmen. ${ }^{46}$

\section{BIBLIOGRAPHIE}

Amselek, P. (Hg.) (1986): Théorie des actes de langage, éthique et droit, Paris: PUF.

Amselek, P. (1987): »Lois juridiques et lois scientifiques«, Droits, Nr. 6.

Amselek, P. (1988): Science et déterminisme, éthique et liberté. Essai sur une fausse antinomie, Paris:

PUF.

Amselek, P. (1997): »La part de la science dans l'activité des juristes«, Recueil Dallos (verfügbar unter http://paul-amselek.com/textes/science_activite_juristes.pdf).

Austin, J. L. (1972 [1962]): Zur Theorie der Sprechakte (How to do things with words), deutsche Bearbeitung von Eike von Savigny, Stuttgart: Reclam.

Avril, P. (1997): Les conventions de la Constitution, Paris: PUF.

Bachelard, G. (1987 [1938]): Die Bildung des wissenschaftlichen Geistes. Beitrag zu einer Psychoanalyse der objektiven Erkenntnis, übers. von Michael Bischoff, Frankfurt a. M.: Suhrkamp.

Baranger, D. (2009): »Le piège du droit constitutionnel«, Juspoliticum, Dezember, Nr. 3.

Beaud, O. (1999): »Le droit constitutionnel par-delà le texte constitutionnel et la jurisprudence constitutionnelle. À propos d'un ouvrage récent«, N3C, Nr. 6.

Denquin, J.-M. (2008): „Situation présente du constitutionnalisme. Quelques réflexions sur l'idée de démocratie par le droit«, Juspoliticum, Dezember, Nr. 1.

Drago, G. (2010): »La Cour de cassation, juge constitutionnel«, Gazette du Palais, 26. Mai, Nr. 6.

Durkheim, É. (1995 [1895]): Regeln der soziologischen Methode, übersetzt von René König, Frankfurt a. M.: Suhrkamp.

Favoreu, L. (1992): Les cours constitutionnelles, Paris: PUF.

Hume, D. (1928 [1748]): Eine Untersuchung über den menschlichen Verstand, übersetzt von Raoul Richter, Hamburg: Meiner.

Kelsen, H. (1976): Reine Rechtslehre, zweite, vollständig neu bearbeitete und erweiterte Auflage, Wien: Deuticke.

Le Bon, G. (1951 [1895]): Psychologie der Massen, übersetzt von Rudolf Eisler, Stuttgart: Kröner.

Mach, E. (1973 [1883]): Die Mechanik in ihrer Entwickelung historisch-kritisch dargestellt, Darmstadt: Wissenschaftliche Buchgesellschaft.

Magnon, X. (2014): »Retour sur quelques définitions premières en droit constitutionnel: que sont une "juridiction constitutionnelle", une "cour constitutionnelle" et une "cour suprême"? Proposition de définitions modales et fonctionnelles«, in: Long cours: Mélanges en l'honneur de Pierre Bon, Paris: Dalloz. S. 305-322. 
Molfessis, N. (2010): »La jurisprudence supra-constitutionem«, JCP G, 18. Oktober.

Pfersmann, O. (2001): »Le droit comparé comme interprétation et comme théorie du droit«, RIDC, Nr. 2.

Picard, E. (2009): »Le droit comparé est-il du droit?«, Annuaire de l'Institut Michel Villey.

Pinat, C.-S. (2015): Le discours de l'avocat devant la Cour de cassation. Étude de théorie du droit, thèse, Montpellier.

Platon (1991): Politeia, in: Sämtliche Werke, Bd. V, nach der Übersetzung Friedrich Schleiermachers, ergänzt durch Übersetzungen von Franz Susemihl und anderen, Frankfurt a. M.: Insel.

Platon (1991): Timaios, in: Sämtliche Werke, Bd. VIII, nach der Übersetzung Friedrich Schleiermachers, ergänzt durch Übersetzungen von Franz Susemihl und anderen, Frankfurt a. M.: Insel.

Ponthoreau, M.-C. (2007): »Cultures constitutionnelles et comparaison en droit constitutionnel. Contribution à une science du droit constitutionnel«, in: Mélanges en l'honneur de Slobodan Milacic, Brüssel: Bruylant.

Ponthoreau, M.-C. (2010): Droits(s) constitutionnels(s) comparé(s), Paris: Economica.

Popper, K. R. (1973 [1972]): Objektive Erkenntnis: ein evolutionärer Entwurf, übersetzt von Hermann Vetter, Hamburg: Hoffmann und Campe.

Roblot-Troizier, A. (2011): „Le non-renvoi des questions prioritaires de constitutionnalité par le Conseil d'État«, Revue françaises de droit administratif, 27 (4), S. 691-710.

Rousseau, D. (1996): La justice constitutionnelle en Europe, 2. Aufl., Paris: Montchrestien.

Rousseau, D. (2010a): »Toujours >Vive la QPC«, oui!«, Gazette du Palais, 27. Mai.

Rousseau, D. (2010b): »L'art italien au Conseil constitutionnel: les décisions des 6 et 14 octobre 2010«, Gazette du Palais, 21. Oktober.

Rousseau, D. (2013) Droit du contentieux constitutionnel, 10. Aufl., Paris: Montchrestien.

Salles, S. (2015): Le conséquentialisme dans la jurisprudence du Conseil constitutionnel, Thèse, Montpellier.

Samuel, G. (2006): »Droit comparé et théorie du droit«, Revue interdisciplinaire d'études juridiques, Nr. 57, S. 1-35.

Schopenhauer, A. (1994/95 [1819/1844]): Die Welt als Wille und Vorstellung, in: Sämtliche Werke, Band 1 und 2, Frankfurt a. M.: Suhrkamp.

Searle, J. R. (1971 [1969]): Sprechakte. Ein sprachphilosophischer Essay, übersetzt von R. und R. Wiggershaus, Frankfurt a.M.: Suhrkamp.

Troper, M. (1994a): Pour une théorie juridique de l'État, Paris: PUF.

Troper, M. (1994b): »Entre science et dogmatique, la voie étroite de la neutralité«, in: Amselek, P. (Hg.): Théorie du droit et science, Paris: PUF, S. 310-325.

Troper, M. (1995): »La liberté d'interprétation du juge constitutionnel«, in: Amselek, P. (Hg.): Interprétation et droit, Brüssel: Bruylant, S. 235 ff.

Troper, M. (2001): La théorie du droit, le droit, l'État, Paris: PUF. 
Troper, M. (2011): »Les concepts juridiques et l'histoire«, in: ders.: Le droit et la nécessité, Paris: PUF.

Troper, M. / Champeil-Desplats, V. / Grzegorczyk C. (Hg.) (2005): Théorie des contraintes juridiques, Brüssel: Bruylant-LGDJ.

Viala, A. (1999): Les réserves d'interprétation dans la jurisprudence du Conseil constitutionnel, Paris: LGDJ.

\section{NOTES}

1. Staatsrat, Urteil vom 13. Dezember 1884, Cadot, Slg. P. 1158, Schlussanträge Jagerschmidt; Sirey (1892), S. 3 (17), Anmerkung von M. Hauriou.

2. Avril (1997).

3. In einer Rezension zu Pierre Avrils Werk würdigte Olivier Beaud dessen Vorgehensweise. Diese hatte seiner Ansicht nach den Vorteil, »den monopolistischen Anspruch der Verfassungsrechtler in Frage zu stellen, die nur noch auf die Rechtsprechung des Verfassungsrates schwören«. Beaud ruft anschließend dazu auf, dieser "Kurzsichtigkeit« der Verfassungsrechtslehre ein Ende zu setzen, welche die Rechtsprechung des Verfassungsrates zum "Alpha und Omega» des Verfassungsrechts erhebt (Beaud [1999], S. 68). Diese Form des epistemologischen Widerstands sollte später zu konkreten Initiativen führen, unter anderem insbesondere zur Gründung, im Jahr 2008, der Zeitschrift Juspoliticum, die in sehr gelungener Weise der vorherrschenden Zeitströmung im Verfassungsrecht eine »Alternativkultur« entgegensetzt, welche den rein politikwissenschaftlichen Aspekt der wissenschaftlichen Disziplin bevorzugt (und dessen guten Ruf wiederherzustellen sucht).

4. Denis Baranger hatte die Verfassungsrechtler bereits kurz vor dem Inkrafttreten des neuen Instituts der vorrangigen Frage zur Verfassungsmäßigkeit vor der, in seinen Worten, »Falle des Verfassungsrechts« gewarnt und stattdessen als Gegenmittel einer die historische Dimension der Rechtsdisziplin betonenden Vorgehensweise den Vorzug gegeben, Baranger (2009), Nr. 3; vgl. auch Denquin (2008), Nr. 1.

5. Diese Ansicht vertritt jedenfalls Paul Amselek, der keinerlei Lob übrig hat für den Anspruch der Juristen, ihre Disziplin zu einer wissenschaftlichen Disziplin zu erheben. Er schreibt: „Genau darin liegt die Hauptaufgabe der Juristischen Fakultäten, nämlich juristisches Handwerkswissen zu bilden und weiterzugeben, wohingegen wissenschaftlichen Erkenntnissen bedauerlicherweise bloß eine Nebenrolle zukommt. Dies schmälert jedoch in keiner Weise das mit dieser Tätigkeit einhergehende grundlegende gesellschaftliche Interesse, denn dieses juristische Handwerk ist die Grundlage für juristische Erfahrung in unseren Gesellschaften, es erlaubt diesen, weiter zu bestehen, sich an die nächsten Generationen weiterzugeben und durch diese voranzuschreiten; dies stellt bereits für sich genommen ein Verdienst dar und bedarf sicherlich keiner Verbürgung durch das Etikett >Wissenschaft«", Amselek (1997), S. 337.

6. Bachelard (1987 [1938]).

7. Dieser Begriff geht auf den französischen Soziologen Gustave Le Bon zurück; s. Le Bon (1951 [1895]).

8. Kelsen (1976 [1960]), S. 3.

9. Die nicht-normativen sozialen Gegebenheiten, mit denen sich der Soziologe auseinandersetzt, sind genauso stumm wie die natürlichen Gegebenheiten. Während die den Gegenstand der Rechtswissenschaften bildende Welt aus Normgehalten besteht, die Werte vorschreiben, und zwar unter Verwendung derselben semantischen Struktur, auf die der Jurist zurückgreift, wenn er diese normativen Aussagen beschreibt, besteht die Welt, mit der sich der Soziologe befasst und sei sie auch Trägerin von Werten -, lediglich aus einer Gesamtheit nicht-normativer 
Gegebenheiten oder Ereignisse, die sich nicht als solche bezeichnen und manchmal über den Willen der Menschen hinauswachsen, deren geistiges Kind sie sind. Wenn Durkheim schrieb, man müsse »soziale Gegebenheiten wie Dinge behandeln«, vertrat er damit implizit die Auffassung, dass die Dinge, welche die empirischen Wissenschaften zu ihrem Gegenstand erheben, stumm seien und es daher geboten sei, sie im Lichte des Kausalitätsprinzips zu untersuchen, des Denkinstruments, das für die konstruktive Dimension des wissenschaftlichen Denkens am bezeichnendsten sei, s. Durkheim (1995 [1895]).

10. Kelsen (1976 [1960]), S. 3.

11. Viala (1999).

12. Salles (2015).

13. Troper (2001), S. VII. Indem er näher darlegt, dass die Rechtssprache sogar dem Gehalt der theoretischen Aussage entgegenwirken kann, spielt Troper hier auf die im Übrigen sehr häufigen Fälle an, in denen der Richter durch die Verwendung von Formulierungen wie »es folgt aus ...« oder »in Erwägung dessen, dass ...« erkennen lässt, dass er das Gesetz auf mechanische Weise auslegt, ohne dessen Wortlaut zu verraten, womit er gleichzeitig die theoretische Annahme widerlegt, gemäß welcher »Auslegung eine Tätigkeit des Willens« sei. Nach Tropers Ansicht verraten die vom Richter verwendeten Formulierungen hingegen, dass Auslegung für diesen eine Tätigkeit der Erkenntnis ist. Weit davon entfernt, die Richtigkeit des theoretischen Diskurses zu widerlegen, stellt diese Diskrepanz zwischen dem Gehalt der theoretischen Aussagen und dem Gehalt der normativen Aussagen gerade das dar, was die Rechtstheorie interessant macht: Um die Herausforderung der Falsifizierbarkeit zu meistern, vor die der richterliche Diskurs den Juristen stellt, wird dieser die Diskrepanz mit der Unbedarftheit des Richters zu erklären suchen, oder damit, dass der Richter die Illusion und die Legende seiner Folgsamkeit gegenüber dem Gesetzgeber aufrechterhalten wolle, um auf diese Weise seine Normsetzungsmacht zu verschleiern und seine Legitimität zu bewahren. Hier zeigt sich, wie der Jurist, indem er auf eine theoretische Auseinandersetzung und die Konstitution seines wissenschaftlichen Gegenstandes verzichtet, dem Test durch den von Karl Popper so bezeichneten Falsifikationismus ausweicht, der für Popper die Wissenschaftlichkeit eines Gedankenganges auszeichnet; Popper (1973 [1972]).

14. Platon, Politeia, Buch VII, S. 509 ff.

15. Ponthoreau (2007), S. 219; Ponthoreau (2010).

16. Favoreu (1992); für eine ähnliche rechtstheoretische Anstrengung durch andere, für die moderne Hinwendung zum Rechtsprechungskommentar im Verfassungsrecht repräsentative Autoren vgl. Rousseau (1996), S. 13 f.; Rousseau (2013), S. 16 f.; Magnon (2014), S. 305.

17. Carré de Malberg hat seine Grundsätze zur Staatstheorie auf der Grundlage einer Untersuchung zu den ideologischen Vorannahmen der Französischen Revolution ausgearbeitet, Prinzipien, die - so Michel Troper - »auch für andere Länder und andere Zeitalter gültig« seien, Troper (1994a), S. 16.

18. Kelsen (1976), S. 3.

19. Für die Beziehung zwischen Rechtsvergleichung und Rechtstheorie vgl. Pfersmann (2001),

S. 275; Samuel (2006), S. 32; Picard (2009), S. 173.

20. Schopenhauer (1994/95).

21. Platon (1991), Timaios, 37d, S. 257.

22. Troper (2011), S. 265.

23. Amselek (1987), S. 131.

24. Ebd.

25. Kelsen (1976), S. 78.

26. Hume (1928 [1748]), S. 90 ff.

27. Amselek (1988), S. 45. 
28. Die Natur »ist nur einmal da«, schrieb im 19. Jahrhundert der Physiker Ernst Mach, der einen starken Einfluss auf den sehr empiristisch geprägten Wiener Kreis ausgeübt hat; Mach (1973 [1883]), S. 459.

29. Für Kelsen ist die Zurechnung in der Welt der Normen das Gegenstück zur Kausalität in der Welt der Naturgesetze: „Die Analogie besteht darin, dass das in Rede stehende Prinzip in den Rechtssätzen eine ganz ähnliche Funktion hat wie das Kausalitätsprinzip in den Naturgesetzen, mit denen die Naturwissenschaft ihren Gegenstand beschreibt«, Kelsen (1976), S. 80. Wie Kelsen kurz darauf weiter ausführt, beschränkt sich die Ähnlichkeit darauf, dass die Zurechnung und das Kausalitätsprinzip beide Verknüpfungen herstellen. Doch die Ähnlichkeit geht nicht weiter, und beide Grundsätze divergieren in Bezug auf das Wesen einer solchen Verknüpfung: die Zurechnung durch die Norm ist eine Verknüpfung, die einem auslösenden Sachverhalt eine normative Folge nach der Hypothese zuordnet »Wenn A ist, soll B sein«, während die Kausalitätsverknüpfung durch das Naturgesetz nach dem einer deterministischen Vorgehensweise erfolgt und eine Wirkung mit einer Ursache verbindet nach dem Schema »Wenn A ist, wird B sein «.

30. Zu dieser begrifflichen Unterscheidung vgl. Troper (2001), S. 12.

31. Kelsen (1976), S. 353 f.

32. Verfassungsrat, Entscheidung Nr. 98-408 DC vom 22. Januar 1999 - Vertrag über das Statut des Internationalen Strafgerichtshofes, amtl. Slg., S. 29.

33. Kassationsgerichtshof (Plenum), Urteil vom 10. Oktober 2001, Rs. Nr. 01-84922, Breisacher.

34. In dieser Debatte standen sich zwei Auffassungen gegenüber, die, jede für sich, Ausdruck einer Positionierung waren, welche das Neutralitätsgebot der Wissenschaft übertraten. Manche Autoren vertraten die Ansicht, die umstrittene Verfassungsbestimmung nehme den Staatspräsidenten auch dann von der Unterwerfung unter die Gerichtsbarkeit aus, wenn er nicht in Verbindung mit der Ausübung des Präsidentenamtes gehandelt habe (so Georges Vedel oder auch Guy Carcassonne), während andere den entgegengesetzten Standpunkt vertraten und im Staatspräsidenten außerhalb dessen Amtsausübung einen gewöhnlichen Bürger sahen, gegen den ein Verfahren vor den ordentlichen Gerichten zulässig sei (so Dominique Rousseau oder auch Olivier Duhamel). Zwar emanzipierten sich diese Darlegungen aus der Rechtslehre vom Wortlaut des Artikels 68 der Verfassung, doch führte der gewollte Charakter dieser Darlegungen, in Ermangelung einer (damals noch nicht ergangenen) Rechtsprechung des Verfassungsrates oder des Kassationsgerichtshofes, deren Autoren dazu, die Grenzen des Neutralitätsgebotes zu überschreiten, denen eine Wissenschaft unterworfen ist. Für die Unterscheidung zwischen Rechtslehre und Rechtswissenschaft vgl. insbesondere die Dissertation von Cathie-Sophie Pinat, Le discours de l'avocat devant la Cour de cassation. Étude et théorie du droit, Universität Montpellier, 2015.

35. Die Unterscheidung zwischen Semantik und Pragmatik geht auf den Semiotiker Charles W. Morris (1901-1979) zurück. Während die Semantik sich mit der Bedeutung einer Aussage beschäftigt, befasst sich die Pragmatik damit, was der Sprecher tut, wenn er die Aussage tätigt: Die pragmatische Dimension hinter der Aussage, durch die der Professor sagt, »das Wetter ist schön«, erlaubt es dessen Studentin zu verstehen, dass er ihr den Hof macht, da man aufgrund des schönen Wetters ja einen Kaffee trinken gehen könnte. Die umfassendsten Ausführungen zur pragmatischen Dimension der Sprache sind allerdings diejenigen des Oxforder Philosophen aus den 1950er Jahren, John L. Austin, sowie von dessen Schüler, John R. Searle. Vgl. Austin (1972 [1962]); Searle (1971 [1969]).

36. Vgl. z. B. Rousseau (2010a), S. 1545, insb. S. 1547; Drago (2010), S. 1438; Roblot-Troizier (2011), S. 691.

37. »Wissenschaftliche Erkenntnisse«, schreibt Michel Troper, »können nicht nur über den Grundsatz der Neutralität definiert werden. Sie müssen eine Erkenntnis bereithalten, mit 
anderen Worten: etwas zutage bringen, das nicht unmittelbar ersichtlich ist«, Troper (1994b), S. 310 und insb. S. 321.

38. Was der Sprecher tut, wenn er eine Aussage tätigt, ist nur außerhalb des Ausspruches erkennbar. Weit mehr als der Sinngehalt der Aussage selbst gibt die Stellung des Sprechers gegenüber seinem Gesprächspartner Aufschluss über das Wesen der Aussage und ob sie z. B. eine Feststellung, eine Anweisung, ein Versprechen, ein Geständnis usw. beinhaltet. Vgl. Amselek (1986).

39. Troper et al. (2005).

40. Troper (1995), S. 235.

41. Baranger (2009).

42. Verfassungsrat, Entscheidung Nr. 2010-39 QPC vom 6. Oktober 2010, amtl. Slg., S. 264; Verfassungsrat, Entscheidung Nr. 2010-52 QPC vom 14. Oktober 2010, amtl. Slg., S. 283.

43. Rousseau (2010b), S. 12-15.

44. Kassationsgerichtshof (Strafsenat), Urteil vom 19. Mai 2010, Rs. Nr. 09-83328, 09-87307 und 09-82582.

45. Molfessis (2010), S. 1955.

46.

INDEX

Schlüsselwörter : Verfassungsrecht, Verfassungswissenschaft, Rechtsprechung, Methodik Mots-clés : droit constitutionnel, science constitutionnelle, jurisprudence, méthode

\section{AUTEURS}

\section{ALEXANDRE VIALA}

Alexandre Viala ist Professor für öffentliches Recht an der Universität Montpellier. Nähere Informationen finden Sie hier. 